\title{
Index anglais
}

\section{English finderlist}

\begin{tabular}{|c|c|c|c|}
\hline abandon & $5.4 .3-3$ & adze & $7.5 .2-3$. \\
\hline abdomen & 1.4.1.2-4. & adze to trim canoes & $7.5 .2-4$ \\
\hline able (be $\sim)$ & $5.6 .4-7$ & Aetobatus narinari & $9.9 .4-1$. \\
\hline abort & $6.1 .3-3$ & affected (be $\sim$ ) & 6.3.1.2-6. \\
\hline abort & $9.3-28$ & affection & 6.3.2.1-3. \\
\hline abortion & $9.3-28$ & affront & $6.8 .1-2$ \\
\hline absent (be $\sim)$ & 6.8.5.3-1. & afire (be $)$ & 7.6.1-1. \\
\hline absent minded & $6.8 .1-18$ & afraid (be $\sim$ ) & 6.3.1.3-3. \\
\hline Abudefduf sordidus & $9.9 .32-1$. & African Pompano & $9.9 .24-5$. \\
\hline abyss & $8.2 .2-14$ & $\operatorname{again}(d o \sim)$ & 7.13.3-3. \\
\hline Acanthocybium solandri & $9.9 .46-4$. & aggravating & 2.4.1.2-7. \\
\hline Acanthurus Achilles & $9.9 .38-5$. & agree & 6.8.2.8-2; \\
\hline Acanthurus guttatus & $9.9 .38-8$. & & 6.8.3.1-1. \\
\hline Acanthurus nigricans & $9.9 .38-4$. & agree (eye gesture) & $5.5 .2-4$ \\
\hline Acanthurus sohal & $9.9 .38-6$. & aground (run ) & 7.5.4.2-1. \\
\hline Acanthurus triostegus & $9.9 .38-3$. & aid & 6.8.3.2-3. \\
\hline Acanthurus & $9.9 .38-7$. & aim at & $7.3 .2-2$ \\
\hline xanthopterus & & albinos & $1.1 .2-7$ \\
\hline accept & $6.2 .3-4 ; 6.5 .4-2$ & Albula glossodonta & $9.9 .8-1$ \\
\hline accompany & $5.2 .4-3 ; 6.8 .5 .2-1$ & Alectis ciliaris & $9.9 .24-5$. \\
\hline accuse & 6.8.4.5-2. & Aleurites moluccana & $10.4 .4-4$ \\
\hline accuse wrongly & 6.8.4.5-3. & alienation & $3.7-3$ \\
\hline acid & $2.1 .1-1$ & alight & 7.6.1-1. \\
\hline acne & $3.2 .1-4$ & almond (tropical ) & $10.3 .5-1$ \\
\hline Adam's apple & $1.3 .9-3$ & Alocasia macrorrhiza & $10.2 .1-4$ \\
\hline add (water) & 7.7.2.4-6. & alone & 6.8.1-14; 6.8.5.1-1. \\
\hline admire & 2.4.2.1-3; 5.5.2-5. & Aluterus scriptus & $9.9 .43-1$. \\
\hline adopt & $6.1 .5-5 ; 6.4 .1-10$. & amazed (be ) & 6.3.1.2-4. \\
\hline adore (God) & 6.6.4.1-13. & ambarella & $10.3 .5-5$ \\
\hline adorn, adorned & $4.4-4$ & ammoniacal smell & 2.4.2.2-6. \\
\hline adult & $6.1 .6-10$. & Amomum cevuga & $10.4 .8-4$ \\
\hline adultery & $6.1 .2-4 ; 6.1 .2-5$ & amputate & $3.6-8$ \\
\hline advice & 6.8.3.2-1. & anaemia & $3.4-1$ \\
\hline advise & 6.8.3.2-2. & & \\
\hline
\end{tabular}




\begin{tabular}{|c|c|c|c|}
\hline \multirow{2}{*}{$\begin{array}{l}\text { Anampses } \\
\text { caeruleopunctatus }\end{array}$} & \multirow[t]{2}{*}{$9.9 .33-9}$. & Arothron hispidus & $9.9 .45-3$. \\
\hline & & Arothron meleagris & $9.9 .45-2$ \\
\hline Anas poecilorhyncha & $9.1 .3-7$. & Arothron stellatus & $9.9 .45-1$. \\
\hline Anas superciliosa & $9.1 .3-7$. & arouse & 6.8.4.3-3. \\
\hline ancestors & $6.4 .1-5 ; 6.4 .3-4$ & arrange & $5.4 .21 .1-2$ \\
\hline anchor & 7.5.4.3-4. & arrange (hot stones in & 7.7.3.1-4. \\
\hline \multirow{2}{*}{ anger, angry } & 6.3.2.3-1; & the oven) & \\
\hline & 6.8.4.3-2. & arrive & $5.2 .8-1 ; 5.2 .8-2$ \\
\hline angle (fishing) & 7.4.3.1-3. & arrow & $7.3 .1-8$. \\
\hline angle (inside) & $7.1 .6-10$. & artery & $1.2-7$ \\
\hline angle (outside) & $7.1 .6-11$ & articulation (bone $\sim$ ) & $1.2-4$ \\
\hline angry (make s.o. ) & 6.3.2.3-2. & Artocarpus altilis & $10.2 .3-1$ \\
\hline animal & $9.3-1$ & Artocarpus altilis & $7.2 .6-3$ \\
\hline animal (young of $\sim$ ) & $9.3-30$ & ashamed (be $\sim$ ) & 6.3.1.4-1. \\
\hline ankle & $1.5 .2-6$ & ashes & $7.6 .2-6$. \\
\hline Annona muricata & $10.3 .6-5$ & ask & 6.8.2.3-1. \\
\hline Annona squamosa L. & $10.3 .6-4$. & ask a question & 6.8.2.3-2. \\
\hline announce & $6.2 .5-2$ & aspect & $6.8 .1-13$ \\
\hline annoy & 6.8.4.1-2. & assist & 6.8.3.2-3. \\
\hline annoyed (be $\sim$ ) & 6.3.1.4-1. & asthma & $3.1-8$ \\
\hline anoint with oil & $4.4-6$ & astringent & $2.1 .1-6$. \\
\hline Anous minutus & $9.1 .2-15$. & attach & 5.4.13-1; 5.4.13-5; \\
\hline Anous stolidus & $9.1 .2-14$. & & $5.4 .13-8$ \\
\hline \multirow[t]{2}{*}{ answer } & 6.8.2.3-3; & attach (loose) & $5.4 .13-6$. \\
\hline & $6.8 .2 .3-4$ & attack (a prey) & 7.4.3.2-1. \\
\hline ant & $9.2-16$ & attain & $5.2 .8-1$ \\
\hline anthrax & $3.2 .2-4$ & attention & $6.8 .1-16$. \\
\hline anus & 1.4.1.3-8. & attractive & $1.1 .2-1$ \\
\hline anxious (be $\sim$ ) & 6.3.1.4-2. & Audubon's Shearwater & $9.1 .2-4$. \\
\hline Apherus furcatus & $9.9 .25-1$. & Aulostomus chinensis & $9.9 .14-1$. \\
\hline Apogon doryssa & $9.9 .20-1$. & aunt & $6.4 .3-3$ \\
\hline appear & $5.1 .7-1$ & avenge & 6.8.4.4-9. \\
\hline appear (in the air) & $5.1 .7-4$ & avoid & $5.2 .13-2 ; 5.2 .9-5$ \\
\hline appear on the surface & $7.5 .5-6$. & awake (stay ) & $2.3 .2-3$ \\
\hline appease & 2.4.1.2-4. & awful & $6.3 \cdot 1.3-4$ \\
\hline applaud & $5.4 .1-5$ & axe & $7.2 .1-4$. \\
\hline apple (custard- ) & $10.3 .6-5$ & Axilspot Hogfish & $9.9 .33-1$. \\
\hline apple (Malay ) & $10.3 .5-5$. & baby & $6.1 .6-1 ; 6.1 .6-2$. \\
\hline apple (sugar ) & $10.3 .6-4$. & bachelor & $6.1 .1-6$. \\
\hline approach & $5.2 .10-1$ & back & $1.4 .1 .2-2$ \\
\hline Aprion virescens & $9.9 .25-2$. & back (of the house) & $7.1 .6-9$. \\
\hline Architectonicidae & $9.6 .2-10$. & back molar & $1.3 .8-7$ \\
\hline Arcidae & $9.6 .2-13$. & bad & $6.8 .1-19$. \\
\hline argue over & 6.8.4.4-1. & bad (gone $\sim$ ) (fish, fruit) & 7.7.4.7-3. \\
\hline arm & $1.5 .1-1$ & bad luck & $7.4-2$ \\
\hline armpit & 1.4.1.1-3. & bad taste & $2.1 .1-7$ \\
\hline aroma & $4.4-5$ & badly cut hair & $1.3 .2-8$ \\
\hline
\end{tabular}




\begin{tabular}{|c|c|c|c|}
\hline bag & 7.9.3-7. & bay & $8.2 .2-5$ \\
\hline bail out & 7.5.3.1-13. & be born & $6.1 .4-2$ \\
\hline bailer & 7.5.3.1-13. & beach & $8.2 .2-10$ \\
\hline \multirow[t]{2}{*}{ bait } & 7.4.3.2-2; & beach almond & $10.3 .5-1$ \\
\hline & 7.4.3.2-4. & Beach Morning-glory & 10.4.7-1. \\
\hline bake again & 7.7.3.2-2. & Beach Naupaka & $10.4 .6-2$ \\
\hline bald & $1.1 .2-8$. & beak (turtle) & $9.10-8$ \\
\hline Balistapus undulatus & $9.9 .42-5$. & beam & 7.1.5.2-2. \\
\hline Balistoides viridescens & $9.9 .42-6$. & beam (wood) & $7.1 .3-6$ \\
\hline ball & $7.12 .2-5$ & bean & $10.3 .1-2$ \\
\hline bamboo & 7.9.2-3; 10.4.8-12. & bear fruit & $7.2 .5-4 ; 10.1 .3-2$ \\
\hline bamboo (recipient) & 7.7.1-16. & beard & $1.3 .3-5$ \\
\hline banana & $10.2 .2-2$ & Bearded Brotula & $9.9 .12-1$ \\
\hline banana & 7.7.4.3-2. & beat & $1.4 .2-2$ \\
\hline banana (dry leaf) & 10.1.1-11. & beat the dust from & $7.1 .8-4$. \\
\hline banana (ripe spotted $\sim$ ) & $7.2 .6-4$ & beat the windward & 7.5.4.1-11. \\
\hline banana plant & 7.7.4.3-2. & beat to death & $5.4 .28-4$ \\
\hline bananas (cluster of $\sim$ ) & $10.2 .2-4$ & beautiful & $1.1 .2-1$ \\
\hline bananas (dried ) & 7.7.4.5-2. & bed & 7.1.7-9. \\
\hline \multirow{2}{*}{$\begin{array}{l}\text { bananas (small bunch } \\
\text { of } \sim \text { ) }\end{array}$} & \multirow[t]{2}{*}{$10.2 .2-3$} & bedroom & 7.1.6-6. \\
\hline & & bee & $9.2-5$ \\
\hline bandage (a sore) & $3.6-7$. & beef & $9.3-25$ \\
\hline bang with fist & $5.4 .28-6$. & beefy & $1.1 .2-3$ \\
\hline banish & $6.8 .4 .9-4$ & beg & 6.8.2.6-4. \\
\hline banyan roots & $10.1 .1-8$ & begin & $5.6 .2-5$ \\
\hline baptise & $6.6 .4 .1-5$ & belch & $2.1 .3-1 ; 2.1 .3-2$ \\
\hline \multirow{2}{*}{$\begin{array}{l}\text { barb (of a hook, an } \\
\text { arrow, a harpoon) }\end{array}$} & \multirow[t]{2}{*}{ 7.4.2-2. } & belittle & 6.8.4.5-4. \\
\hline & & bellow & $9.3-26$. \\
\hline barbels & $9.9 .1-14$ & belly & 1.4.1.2-4. \\
\hline bark & $10.1 .1-4$ & belly (turtle) & $9.10-4$ \\
\hline bark & $9.3-4$ & belt & $4.1-11$. \\
\hline Barred Filefish & $9.9 .43-2$ & bench & 7.1.7-6. \\
\hline Barred Flagtail & $9.9 .21-1$ & bend (s.th.) & $5.4 .11-5 ; 5.4 .20-1$ \\
\hline Barred Jack & $9.9 .24-2$ & bend (with too much & $7.2 .5-11$ \\
\hline Barred Thicklip & $9.9 .33-16$ & fruit) & \\
\hline barrel (large $\sim$ ) & 7.7.4.6-7. & bend (wood) & $7.11 .4-8$. \\
\hline Barringtonia asiatica & $10.4 .3-5$ & bend down & $5.4 .11-6$ \\
\hline basil (local ) & $10.3 .1-3$ & bent & $5.1 .5-3 ; 5.1 .5-1$. \\
\hline \multirow[t]{2}{*}{ basket } & \multirow{2}{*}{$\begin{array}{l}\text { 7.9.3-1; 7.9.3-4; } \\
\quad 7.9 .3-5 ; 7.9 .3-6 .\end{array}$} & bequest & 6.5.3.1-2. \\
\hline & & berth & 7.5.4.3-1. \\
\hline basket handle & 7.9.3-2. & betrothed (be $\sim$ ) & $6.1 .2-6$ \\
\hline bastard & 6.8.4.7-4. & bewitch & 6.6.1-1. \\
\hline Batatas edulis & $10.2 .1-7 ; 10.2 .2-1$ & Bible & $6.6 .4 .5-2$ \\
\hline bathe & $7.5 .5-3$ & Bicoloured Cleaner & $9.9 .33-22$ \\
\hline bathroom & 7.1.6-4. & Wrasse & \\
\hline battle & 6.8.4.9-2. & big & $1.1 .1-2 ; 1.1 .3-1$. \\
\hline bawdy song & 6.7.3.2-2. & Bigeye Emperor & $9.9 .27-1$ \\
\hline
\end{tabular}




\begin{tabular}{|c|c|c|c|}
\hline Bigeye Jack & $9.9 .24-8$. & Bluelined Snapper & $9.9 .25-3$. \\
\hline bile & $1.4 .2-6$ & Blue-spotted Grouper & 9.9.17-1. \\
\hline \multirow{2}{*}{$\begin{array}{l}\text { bind with fine coconut } \\
\text { fibres }\end{array}$} & \multirow[t]{2}{*}{ 7.5.2-1. } & Bluespotted Wrasse & $9.9 .33-9$. \\
\hline & & blunt & $7.12 .5-6$. \\
\hline bird & $9.1 .1-1$ & boar & $9.3-16$ \\
\hline bird (young ) & $9.1 .1-5$ & boat frame & 7.5.3.1-6. \\
\hline Bird Wrasse & $9.9 .33-18$. & Bodianus axillaris & $9.9 .33-1$. \\
\hline Birgus latro & $9.8-7$ & body & $1.1-2$. \\
\hline bitter & $2.1 .1-2$ & \multirow[t]{2}{*}{ boil } & \multirow{2}{*}{$\begin{array}{c}3.2 .2-3 ; 7.7 .2 \\
7.7 .3 .2-8\end{array}$} \\
\hline black & 7.12.4-1. & & \\
\hline black & $7.12 .4-2$. & bold & \\
\hline Black Barracuda & $9.9 .35-2$. & bone & $1.2-3$ \\
\hline Black Jack & $9.9 .24-10$ & bone (of fish) & $9.9 .1-6$ \\
\hline Black Noddy & $9.1 .2-15$. & Bonefish & $9.9 .8-1$ \\
\hline Black Triggerfish & $9.9 .42-1$. & bony & $1.1 .4-2$ \\
\hline Black Unicornfish & $9.9 .39-6$. & boom of a canoe & 7.5.3.1-2. \\
\hline Black-Edged Conger & $9.9 .6-10$. & outrigger & \\
\hline Blackfish Squirrelfish & $9.9 .13-1$. & border & $7.1 .1-2$ \\
\hline \multirow{2}{*}{$\begin{array}{l}\text { Blacksaddled } \\
\text { coralgrouper }\end{array}$} & \multirow[t]{2}{*}{$9.9 .17-11}$. & bore & $5.4 .27-3 ; 6.8 .4 .1-2$ \\
\hline & & boredom & 6.3.1.4-5. \\
\hline Blackspot Sergent & $9.9 .32-1$. & borer & $7.11 .1-3$. \\
\hline Blackspot Snapper & $9.9 .25-4$. & born (be $\sim)$ & $6.1 .4-2$ \\
\hline Blacktip Grouper & $9.9 .17-5$. & bother & 6.8.4.1-2. \\
\hline Blacktip Shark & $9.9 .2-2$ & Bothus mancus & $9.9 .41-1$. \\
\hline bladder & $1.4 .2-7$ & Bothus pantherinus & $9.9 .41-2$ \\
\hline blade (of paddle) & 7.5.3.1-10. & bottle & 7.7.1-6. \\
\hline blanket & 7.1.7-2. & bounce & $8.1 .5-3$ \\
\hline blaspheme & 6.8.4.7-2. & boundary mark & 7.1.1-2. \\
\hline blennorrhoea & $3.4-14$. & bow & $5.1 .5-3 ; 7.3 .1-2$. \\
\hline blind & $3.5-3$ & bow (canoe $\sim)$ & 7.5.3.1-4. \\
\hline blink & $5.5 .2-2$ & bow (shoot with $a \sim$ ) & 7.3.1-9. \\
\hline blister & $3.2 .3-3$ & bow (under the weight of & $7.2 .5-11$ \\
\hline block & $5.4 .30-5$ & fruit) & \\
\hline blood & $1.2-8$ & bowed (be $\sim)$ & $5.1 .5-3$ \\
\hline bloom & $10.1 .2-3$. & bowel & $1.4 .2-4$ \\
\hline bloom (in ) & $10.1 .2-6$ & bowl & 7.7.1-1; 7.7.1-4. \\
\hline blossom (in ) & $10.1 .2-6$. & box & $5.4 .28-2$ \\
\hline \multirow{2}{*}{$\begin{array}{l}\text { blossoms (coconut } \\
\text { palm } \sim \text { ) }\end{array}$} & \multirow[t]{2}{*}{$10.4 .2-6$} & box & 7.1.7-1. \\
\hline & & $\operatorname{box}(t o \sim)$ & 6.8.4.4-4. \\
\hline blow & $2.2-3$ & boy & $6.1 .6-6 ; 6.4 .3-11 ;$ \\
\hline blow (wind) & 8.1.6.1-2. & & $6.5 .2-7$ \\
\hline blow o.'s nose & $2.1 .4-2$ & brag & 6.8.4.3-5. \\
\hline blue & $7.12 .4-4$. & braid (to ) & 7.9.1-3. \\
\hline blue (lagoon) & $7.12 .4-5$. & braided hair & $4.2-3$ \\
\hline Bluebarred Parrotfish & $9.9 .34-3$. & brain & $1.3 .1-3$ \\
\hline Bluefin Jack & $9.9 .24-1$ & branch (small ) & $10.1 .1-3$ \\
\hline Blue-gray Noddy & $9.1 .2-17$. & branche & $10.1 .1-2$ \\
\hline
\end{tabular}




\begin{tabular}{|c|c|c|c|}
\hline brand & 7.6.1-6. & Brown unicornfish & $9.9 .39-3$. \\
\hline Brassy Jack & $9.9 .24-7$ & brush (against) & $5.4 .16-5$ \\
\hline brave & $6.8 .1-2$ & buckle (a rod) & 7.7.2.2-4. \\
\hline bread (coconut ) & 7.7.4.4-1. & bud & 10.1.1-7; 10.1.2-1. \\
\hline bread (communion $\sim$ ) & 6.6.4.5-6. & build & $5.6 .1-1 ; 7.1 .3-1$. \\
\hline breadfruit & $10.2 .3-1$ & builder & $7.1 .3-8$. \\
\hline breadfruit (fermented ) & 7.7.4.5-1. & bullet & 7.3.1-7. \\
\hline \multirow{2}{*}{$\begin{array}{l}\text { breadfruit (non edible } \\
\text { central part of } \sim \text { ) }\end{array}$} & \multirow[t]{2}{*}{ 7.7.2.4-10. } & bump & $5.2 .13-1$ \\
\hline & & bump (on the forehead) & $3.2 .2-1$ \\
\hline \multirow{2}{*}{$\begin{array}{l}\text { breadfruit (which falls } \\
\text { before ripening) }\end{array}$} & \multirow[t]{2}{*}{$7.2 .6-3$} & bump into & $5.2 .5-2$ \\
\hline & & bun & $4.2-2$ \\
\hline breadfruit sap & $10.2 .3-2$ & bunch & $10.1 .3-3$ \\
\hline \multirow[t]{2}{*}{ break } & \multirow{2}{*}{$\begin{array}{l}\text { 5.4.22.2-3; } \\
\text { 5.4.29.3-1; } \\
\text { 5.4.29.3-3. }\end{array}$} & $\begin{array}{l}\text { bunch }(\text { small } \sim \text { of } \\
\text { bananas) }\end{array}$ & $10.2 .2-3$ \\
\hline & & bunch of flowers & $10.1 .2-4$ \\
\hline break open & $7.2 .5-1$ & bundle of firewood & $7.11 .3-2$. \\
\hline breast & $\begin{array}{l}\text { 1.4.1.1-1; } \\
\text { 1.4.1.1-4. }\end{array}$ & burn (piles) & 7.6.2-10. \\
\hline breath & $2.2-2$ & burn down & 7.6.2-9. \\
\hline breath (out of $\sim$ ) & $2.2-6$ & burn rubbish & $7.2 .2-6$ \\
\hline breath of wind & $\begin{array}{l}2.2-6 . \\
2.4 .1 .1-6 .\end{array}$ & burn stubble & $7.2 .2-7$ \\
\hline $\begin{array}{l}\text { breath of wind } \\
\text { hreathe }\end{array}$ & & burn-beat & $7.2 .2-6$ \\
\hline breathe & $2.2-1 ; 2.2-4$ & burning & $7.6 .1-1 ; 7.6 .2-12$ \\
\hline $\begin{array}{l}\text { breathe (on emerging } \\
\text { from the water) }\end{array}$ & $7.5 .5-5$ & burnt & 7.6.2-8. \\
\hline breathe (out of water) & $7.5 .5-5$ & burnt (during cooking) & 7.7.3.3-6. \\
\hline breathing & $2.2-2$ & burp & $2.1 .3-1$ \\
\hline breeze & 2.4.1.1-6. & burst out laughing & $2.4 .1 .3-2$. \\
\hline Bridled Triggerfish & $9.9 .42-3$ & bury & $5.4 .7-6$ \\
\hline \multirow{2}{*}{ bring } & 5.4.2.1-1; & bush (the $\sim$ ) & $10.1 .1-6$ \\
\hline & 5.4.2.2-1. & butter (to $\sim)$ & 7.7.2.1-12. \\
\hline bring closer & $5.4 .6-2$ & butterfly & $9.2-10$ \\
\hline Bristle-thighed Curlew & $9.1 .3-2$ & Butterflyfish & $9.9 .30-1$. \\
\hline broad & $1.1 .1-2$ & buttock & 1.4.1.3-7. \\
\hline \multirow[t]{3}{*}{ broken } & 5.4.29.3-2; & button & $4.1-5$. \\
\hline & 7.12.5-3; & cabbage & 10.3.1-1. \\
\hline & $7.12 .5-13$. & cackle & $9.1 .1-17$. \\
\hline brood & $9.1 .1-8$ & calf (leg's $\sim)$ & $1.5 .2-4$ \\
\hline broom & $7.1 .8-2$ & Calidris alba & $9.1 .3-4$ \\
\hline brother (older) & $6.4 .3-14$ & call & 6.8.2.2-2. \\
\hline brother (younger) & $6.4 .3-13$ & call on (s.o.) & 6.8.3.5-3. \\
\hline brother-in-law & $6.4 .4-4$ & calm & $6.1 .8-9 ; 6.8 .3 .4-1$. \\
\hline brother's wife & $6.4 .4-5$ & calm (a child) & $6.1 .5-3$ \\
\hline Brotula multibarbata & $9.9 .12-1$. & Calophyllum inophyllum & $10.4 .3-4$ \\
\hline Broussonetia papyrifera & $10.4 .3-2$ & can & $5.6 .4-6$ \\
\hline Brown Booby & $9.1 .2-8$ & candleberry tree & $10.4 .4-4$ \\
\hline Brown Noddy & $9.1 .2-14$. & canoe & 7.5.1-2. \\
\hline brown sea-urchin & $9.12-3$. & Cantherhines dumerilii & $9.9 .43-2$ \\
\hline (edible) & & cap & 7.7.1-7. \\
\hline
\end{tabular}


cape

capsize

Carangoides ferdau

Carangoides orthogrammus

Caranx ignobilis

Caranx lugubris

Caranx melampygus

Caranx papuensis

Caranx sexfasciatus

carapace

carbuncle

Carcharhinus albimarginatus

Carcharhinus amblyrhynchos

Carcharhinus longimanus

Carcharhinus melanopterus

Cardiidae

Cardisoma carnifex

carelessness

caress

Carica papaya

carpenter

carpet

Carpilius maculatus

carry (on back)

carry (on hip)

carry (on shoulder)

carry (pole for $\sim$ ing
bananas)

carry off

carve

carve

cask

Cassidae

cast

castrate

Casuarina equisetifolia

cat

catapult

cataract

catch

catch (an insect)
8.2.2-6

7.5.4.2-6.

9.9.24-2.

9.9.24-3.

9.9.24-6.

9.9.24-10.

9.9.24-1.

9.9.24-7.

9.9.24-8.

9.10-2.

3.2.2-4.

9.9.2-5.

9.9.2-3.

$9.9 .2-4$.

9.9.2-2.

9.6.2-16.

$9.8-4$.

6.8.1-18.

5.4.1-4; 5.4.16-2.

10.3.5-2.

$7.1 .3-8$.

7.1.7-7.

9.8-3.

5.4.2.1-4.

5.4.2.1-6.

5.4.2.1-2;

5.4.2.1-3;

5.4.2.1-5.

7.2.1-2.

5.4.2.2-2.

7.11.4-9.

7.7.2.3-6.

7.7.4.6-7.

9.6.2-11.

5.4.25-1.

9.3-17.

10.4.4-6.

9.3-8.

7.3.1-3.

$3.3-2$.

5.4.2.3-6; 7.4.6-1.

$5.4 .16-6$. catch (fruit)

catch (in midair)

catch (river shrimps)

with net

catch (with slipknot)

catch fish with o.'s hand in holes in rocks

caterpillar

catholic

caulk

cause to disappear

cavity (tooth $\sim$ )

cease

ceiling

cement house

cemetery

centipede

central vein and

foot-stalk of coconut palm

Cephalopholis argus

Cephalopholis

sexmaculata

Cephalopholis urodeta

Ceratopogonidae

Cerithiidae

Chaetodontidae)

chain

chair

Chameleon Grouper

change

change direction

Chanos chanos

character

charcoal

charm

Charonia tritonis

charred

chase

chat, chatter

chatterbox

Checkerboard Wrasse

cheek

cheep

Cheilinus chlorourus

Cheilinus oxycephalus
7.2.5-2.

5.4.1-2; 5.4.2.3-8.

7.4.1-4.

5.4.13-10.

7.4.6-6.

9.2-2.

6.6.4.1-8.

5.4.30-5.

5.4.29.5-3.

1.3.8-4.

5.2.15-2.

7.1.5.2-5.

7.1.4-4.

6.1.8-5.

9.2-19.

10.4.2-4.

9.9.17-1.

9.9.17-2.

9.9.17-3.

9.2-9.

9.6.2-3.

9.9.30-1.

4.3-3.

7.1.7-6.

9.9.17-10.

5.4.19-4.

5.2.7-2.

9.9.9-1.

6.8.1-13.

7.6.2-2.

6.6.1-1.

9.6.2-8.

7.6.2-8.

5.2.4-4.

6.8.2.1-1;

6.8.2.9-1.

6.8.2.1-2.

9.9.33-12.

1.3.3-3.

9.1.1-18.

9.9.33-4.

9.9.33-6. 


\begin{tabular}{|c|c|c|c|}
\hline Cheilinus trilobatus & $9.9 .33-3$. & clear water & 7.7.4.6-4. \\
\hline Cheilinus undulatus & $9.9 .33-2$. & clever & $5.6 .4-1$ \\
\hline Cheilio inermis & $9.9 .33-17$ & cliff & $8.2 .2-3$ \\
\hline Chelonia mydas & $9.10-1$ & climb down & $5.2 .2-2$ \\
\hline cherish & 6.3.2.4-3. & climb up & $5.2 .2-1 ; 5.2 .2-3$ \\
\hline chest & 1.4.1.1-1. & climbing plant & 10.4.7-2. \\
\hline chestnut & $10.3 .5-3$. & clitoris & 1.4.1.3-5. \\
\hline chestnut (Polynesian ) & 7.7.4.3-4. & close (door) & $5.4 .30-3$ \\
\hline chew & $2.1 .2-5$ & close (eyes) & 5.5.2.-3. \\
\hline chick & $9.1 .1-5$ & cloth material & $4.1-2$ \\
\hline chickenpox & $3.4-12$ & clothes peg & $7.8-8$ \\
\hline chief & $6.5 .2-2 ; 6.5 .2-5$ & clothing & $4.1-2$ \\
\hline chignon & $4.2-2$ & clothing (bark-made ) & $7.10-8$ \\
\hline child & $6.1 .6-6 ; 6.1 .6-7$. & (tapa) & \\
\hline chili & $10.3 .1-4$ & cloud & $8.1 .4-1$. \\
\hline chin & $1.3 .9-1$ & cloudless sky & $8.1 .1-8$ \\
\hline chirp & $9.1 .1-18$. & cloudy (water) & $8.3 .1-5$ \\
\hline chisel & $7.11 .1-7$. & Clown Coris & $9.9 .33-10$. \\
\hline Chiseltooth Wrasse & $9.9 .33-19$ & Clown Coris & $9.9 .33-10$. \\
\hline Chlorurus microrhinos & $9.9 .34-1$. & club foot & \\
\hline choke & $2.2-5$ & cluster (of fruit) & 10.1.3-3. \\
\hline choke (with food) & $2.1 .3-4 ; 2.1 .3-5$. & cluster of bananas & $10.2 .2-4$ \\
\hline choose & $6.5 .2-4$ & coagulated (blood) & $1.2-8$ \\
\hline chop (into small pieces) & 7.7.2.3-5. & Coccoloba uvifera & $10.4 .8-10$ \\
\hline chop up (into small & 7.7.2.3-4. & cock-a-doodle-do & $9.1 .1-15$. \\
\hline pieces) & & cockle & $9.6 .2-16$. \\
\hline christen & 6.6.4.1-5. & cockroach & $9.2-12$ \\
\hline Christian & 6.6.4.1-9. & coconut & 10.3.2-1; 10.3.2-4. \\
\hline Christian name & $6.1 .1-3$ & coconut (coprah of $\sim$ ) & 10.3.2-7. \\
\hline Christmas Shearwater & $9.1 .2-2$ & coconut (fifth stage) & $10.3 .3-5$ \\
\hline church & 6.6.4.2-1. & coconut (first stage) & $10.3 .3-1$. \\
\hline Cigar Wrasse & $9.9 .33-17$. & coconut (fourth stage) & $10.3 .3-4$ \\
\hline circumcise & $6.1 .6-8$ & coconut (grated & 7.7.2.4-11. \\
\hline Cirrhilabrus scottorum & $9.9 .33-8$. & grounds) & \\
\hline Cirrhitus pinnulatus & $9.9 .19-1$. & coconut (hole of the $\sim$ ) & 10.3.2-3. \\
\hline Citrus aurantiacea & $10.3 .4-2$ & coconut (pure $\sim$ milk) & 7.7.2.4-1. \\
\hline clairvoyant & $6.6 .1-2$ & coconut (second stage) & $10.3 .3-2$ \\
\hline clam & $9.6 .2-13$. & coconut (short $\sim$ ) & 10.4.2-10. \\
\hline clap & $5.4 .1-5$ & coconut (sixth stage) & 10.3.3-6. \\
\hline claw & $1.5 .1-10$ & coconut (third stage) & $10.3 .3-3$ \\
\hline claw (bird) & $9.1 .1-4$. & coconut (young $\sim)$ & $10.3 .2-5$ \\
\hline clay & $8.2 .1-3$ & coconut blossoms & $10.4 .2-5$ \\
\hline clean & $4.4-1 ; 4.4-7$. & (sheath of $\sim$ ) & \\
\hline clean water & 7.7.4.6-4. & coconut blossoms (stalk & $10.4 .2-7$ \\
\hline clear & $8.1 .1-8$. & bearing ) & \\
\hline clear ground & $7.2 .2-3$. & coconut bread & 7.7.4.4-1. \\
\hline clear out (room) & $5.4 .25-4$. & Coconut Crab & $9.8-7$ \\
\hline
\end{tabular}




coconut frond (lower
part of $\sim$ )
coconut grater
coconut grove
coconut husk
coconut leaf
coconut milk (drop in $\sim$ )
coconut milk (season
with $\sim$ )
coconut milk cooked
coconut palm
coconut palm (foot-stalk
and central vein)
coconut palm blossoms
coconut palm heart
coconut palm lichen
coconut palms (roots
of $\sim$ )

coconut tree (frond of $\sim$ ) coconut water

Cocos nucifera

coddle

coffee

cold

colic

collapse

collect

collect (by cutting)

collect (money)

Colocasia esculenta)

colour

comb

come

come alongside

come back to mind

come close

come in

come out (plant)

come together

come with

comet

comfort (o.'s sorrow)

Common Fairy Tern

compete

compress (coprah)
10.4.2-3.

7.7.1-2.

$7.2 .3-11$.

10.3.2-6.

$7.9 .2-2$.

7.7.4.4-6.

7.7.2.4-2.

7.7.3.2-3.

10.3.2-1.

10.4.2-4.

10.4.2-6.

10.4.2-9.

10.4.9-2.

10.4.2-8.

10.4.2-1.

10.3.2-2.

10.3.2-1.

6.1.5-4.

10.4.5-2.

2.4.1.1-2;

2.4.1.1-3.

$3.1-6$.

5.2.12-4.

5.4.21.1-1.

7.2.5-8.

6.6.4.4-1.

10.2.1-2.

7.12.4-12.

4.4-2.

5.2.7-1.

5.2.8-2.

6.2.2-5.

5.2.10-1.

5.2.6-1.

7.2.3-3.

6.8.5.1-3.

6.8.5.2-1.

8.1.1-9.

6.8.3.4-2.

9.1.2-16.

6.8.4.3-4;

6.8.4.4-1.

5.4.21.4-4. conceited

conch

condemn

condolence

conduct

cone ginger

cones

confess

confirm

conflict

Conger cinereus

Conidae

conjunctivitis

consecrate

consent

constipated

container

container (water $\sim$ )

container (wooden

flat )

contractions

contradict

convert (to)

Convict Surgeonfish

convulsion

cook (food)

cook (in steam)

cook (in the oven)

cook again (food)

cooked (properly)

cooking place

cool

Cooper Sweeper

copra (coconut)

copulate

coral

coral (branching )

coral rock

corals (dead )

Cordia subcordata

cordyline

Cordyline terminalis

Coris aygula

Coris Gamardi

cork
6.8.4.2-2

9.6.2-8.

6.8.4.8-6;

6.8.4.8-9.

6.1.8-8.

5.2.4-1.

10.4.8-4.

9.6.2-12.

6.6.4.6-1.

6.8.2.8-1.

6.8.4.4-3.

9.9.6-10.

9.6.2-12.

$3.3-1$.

6.6.4.1-3.

5.5.2-4.

2.1.4-9.

7.7.1-1.

7.7.1-5.

7.7.1-19.

6.1.4-4.

6.8.2.8-4.

6.6.4.1-2.

9.9.38-3.

$3.1-11 ; 3.1-5$.

7.7.3.2-5.

7.7.3.2-6.

7.7.3.1-3.

7.7.3.2-2.

7.7.3.3-7.

7.1.6-7.

2.4.1.1-5; 8.1.4-4.

9.9.28-8.

10.3.2-7.

2.4.2.4-1;

2.4.2.4-2.

9.13.2-1; 9.13.2-2.

9.13.2-3.

9.13.2-1.

9.13.2-5.

10.4.4-5.

7.9.2-1.

7.9.2-1.

9.9.33-10.

9.9.33-11.

7.7.1-7. 


\begin{tabular}{|c|c|c|c|}
\hline corner & $7.1 .6-10 ; 7.1 .6-11$. & crumb, crumble & 5.4.18.1-3. \\
\hline Cornetfish & $9.9 .15-1$. & crumble down & $5.2 .12-4$ \\
\hline corpse & $6.1 .7-2$ & crumbs & 7.7.2.3-3. \\
\hline corrugated iron house & 7.1.4-3. & crunch & $2.1 .2-10$ \\
\hline Coryphaena hippurus & $9.9 .46-1$. & crush & 5.4.18.1-1; \\
\hline cough & $3.4-9$ & & 5.4.18.1-2; \\
\hline \multirow[t]{2}{*}{ council } & 6.8.2.4-1; & & 7.7.2.1-11. \\
\hline & 6.8.5.4-2. & crush with a pounder & 7.7.2.1-11. \\
\hline count & $6.2 .6-3$ & cry & $2.4 .1 .3-6$ \\
\hline couple & $5.4 .21 .2-5$ & cry out & $6.8 .2 .2-3$ \\
\hline courage & 6.3.1.3-1. & Culcita grex & $9.12-6$ \\
\hline courageous & $6.8 .1-6$. & cult & 6.6.4.1-4. \\
\hline court-bouillon (cook in & 7.7.3.2-8. & cure & $3.6-1$ \\
\hline$a \sim)$ & & current & $8.3 .1-2$ \\
\hline cove & $8.2 .2-5$ & cushion & 7.1.7-10. \\
\hline \multirow[t]{2}{*}{ cover } & $5.4 .7-5 ; 7.1 .1-4$ & Cushion Star & $9.12-6$ \\
\hline & 7.1.3-4. & Custard Apple & $10.3 .6-5$. \\
\hline covered (to be $\sim$ ) (oven) & 7.7.3.1-5. & cut & 5.4.22.2-3; \\
\hline cowlick & $1.3 .2-7$ & & 5.4.29.2-2; \\
\hline crab & $9.6 .2-19 ; 9.8-1 ;$ & & 5.4.29.2-3; \\
\hline & $9.8-2 ; 9.8-3$ & & 5.4.29.2-4; \\
\hline & $9.8-4 ; 9.8-5$ & & 5.4.29.4-3; \\
\hline & $9.8-6$ & & $7.10-5 ; 7.11 .4-5$. \\
\hline $\operatorname{crab}(\operatorname{coconut} \sim)$ & $9.8-7$ & cut (into pieces) & 7.7.2.3-6. \\
\hline crack & $7.12 .2-1$ & cut (with teeth) & $2.1 .2-6$ \\
\hline cram & 5.4.21.4-3. & cut off & 5.4.29.2-1. \\
\hline crawl & $5.2 .3-2$ & cutting & $7.2 .4-1$ \\
\hline crayfish (freshwater & 7.7.4.4-3. & Cypraecassis rufa & $9.6 .2-11$. \\
\hline $\begin{array}{l}\text { seasoned with coconut } \\
\text { milk) }\end{array}$ & & Cypraeidae & $\begin{array}{l}9.6 .2-5 ; 9.6 .2-6 \\
9.6 .2-7\end{array}$ \\
\hline crazy & $3.7-2$ & Cypselurus simus & $9.9 .11-2$. \\
\hline create & $5.6 .1-3$ & dad & $6.4 .2-2$ \\
\hline creeps (give the $\sim$ ) & 2.4.1.1-4. & Daira perlata & $9.8-2$ \\
\hline Crenimugil crenilabis & $9.9 .36-1$. & damp & $7.12 .5-18$ \\
\hline crest (mountain ) & $8.2 .2-11$ & dance & $6.7 .2-1$ \\
\hline cricket & $9.2-7$ & dance (old $\sim)$ & $6.7 .2-2$ \\
\hline crippled & $3.5-1$ & dandruff & $1.3 .2-9$ \\
\hline crispy (make) & 7.7.3.2-4. & dare & 6.8.4.3-7. \\
\hline crossed woods & 7.5.3.1-3. & dark & $8.1 .1-13$. \\
\hline (supporting the boom) & & dark (very ) & $7.12 .4-2$ \\
\hline crossingroads & $8.2 .2-2$ & darkness & 8.1.1-13. \\
\hline crouch & $5.1 .2-1$ & darn & $7.10-7$ \\
\hline crow & $9.1 .1-14$. & dash at & $5.4 .11-3$ \\
\hline crowbar & $7.2 .1-8 ; 7.2 .2-4$ & daughter & $6.4 .3-12$ \\
\hline Crown Squirrelfish & $9.9 .13-4$. & daughter-in-law & $6.4 .4-7$ \\
\hline crown & $4.3-1$ & dawn & $8.1 .2-1$ \\
\hline crucify & 6.6.4.1-12. & day & $8.1 .3-9$ \\
\hline crumb & $7.2 .3-4$ & day (whole $\sim)$ & $8.1 .3-9$ \\
\hline
\end{tabular}




\begin{tabular}{|c|c|c|c|}
\hline daylight & $8.1 .1-4$ & dimple & $1.3 .3-4$. \\
\hline dazzled (be $\sim$ ) & $3.3-4$ & dimwit & $3.7-1$. \\
\hline dead corals & $9.13 .2-5$ & dining room & $7.1 .6-5$. \\
\hline deaf & $3.5-5$ & dip & 5.4.18.3-2; \\
\hline dearth & $8.1 .3-13$ & & 5.4.18.3-3. \\
\hline debauched (man) & $6.5 .6-2$ & dip (hands) & 5.4.1-9. \\
\hline debt & 6.5.3.1-4. & dip into water & $7.8-4$. \\
\hline debt (paid ) & 6.5.3.1-5. & dirty & $4.4-8 ; 7.7 .4 .7-6$. \\
\hline decayed tooth & $1.3 .8-4$ & disabled & $3.5-1$. \\
\hline deceive & $6.2 .6-6 ; 6.8 .2 .5-1$ & disagree & 6.8.4.4-2; \\
\hline $\operatorname{deck}(\operatorname{ship})$ & 7.5.3.2-1. & & 6.8.4.4-3. \\
\hline decorate & $4.4-4 ; 5.6 .2-1$. & disappear & $5.1 .7-2$. \\
\hline decorate & $7.1 .8-1$ & discord & 6.8.4.4-2. \\
\hline deep & $7.12 .3-2$ & disdainful & 6.8.4.2-1. \\
\hline deepen (a hole) & $5.4 .26-3$. & disembark & $5.2 .8-2$ \\
\hline defeated & 6.8.4.9-6. & disembowel & 7.7.2.3-1. \\
\hline defecate & $2.1 .4-8$ & disgusted & $2.4 .1 .2-2$. \\
\hline defy & $6.8 .4 .3-4$ & distended (cloth) & $4.1-7$. \\
\hline delicious (fish, crab) & 7.7.4.7-1. & distribute & $5.4 .22 .2-4$ \\
\hline delight & 6.3.1.1-3. & disturbed (be $\sim$ ) & 6.3.1.2-1; \\
\hline delighted (be $\sim$ ) & 6.3.1.1-4. & & 6.3.1.2-2. \\
\hline dementia & $3.7-3$. & divide & 5.4.22.2-1. \\
\hline demijohn & 7.7.1-23. & divorce & $6.1 .2-3$ \\
\hline denigrate & $6.8 .4 .5-4$ & dizziness & 2.4.1.2-6. \\
\hline deny & $5.2 .9-5$ & do & $5.6 .1-2$ \\
\hline depart & $5.2 .8-3$. & do again & 7.13.3-3. \\
\hline descend & $5.2 .2-2$ & do twice & $7.13 .3-3$. \\
\hline descendants & $6.4 .1-6$ & dodge & $5.2 .13-2$ \\
\hline desire & $6.3 .2 .2-1 ; 6.2 .3-3$ & $\operatorname{dog}$ & $9.3-2$ \\
\hline \multirow[t]{2}{*}{ despise } & 6.3.2.1-5; & Dogtooth Tuna & $9.9 .46-5$. \\
\hline & 6.8.4.2-3. & dolphinfish & $9.9 .46-1$. \\
\hline dessert (po'e) & 7.7.4.4-4. & domesticated & $9.3-14$ \\
\hline destroy & 5.4.29.5-3. & done (a meal) & 7.7.3.3-4. \\
\hline detain & $5.4 .12-1$ & donkey & $9.3-18$ \\
\hline deviate & $5.2 .9-7$ & door & 7.1.5.1-5. \\
\hline Devil Scorpionfish & $9.9 .16-1$. & door (mobile part) & 7.1.5.1-1. \\
\hline dew & 8.1.4-7. & doorstep & 7.1.5.1-5. \\
\hline diarrhoea & $3.4-5$ & doorway & 7.1.5.1-2. \\
\hline dice & 7.7.2.3-4. & Dot-dash Goatfish & $9.9 .28-3$. \\
\hline dice & 7.7.2.3-5. & double & $7.13 .3-1$ \\
\hline die & 6.1.7-1. & dough (breadfruit or & 7.7.4.4-5. \\
\hline difficult(y) & $6.2 .5-5$ & banana ) & \\
\hline dig & $5.4 .26-1 ; 5.4 .26-2$. & downward slope & $8.2 .2-12$ \\
\hline dig a hole (in the & 7.2.2-1. & drag & $5.4 .10-3 ; 5.4 .10-4$. \\
\hline ground) & & dragline fishing & $7.4 .5-2$ \\
\hline digging stick & 7.2.1-3. & dragonfly & $9.2-11$. \\
\hline dilute (with water) & 7.7.2.4-6. & draught & 8.1.6.1-4. \\
\hline
\end{tabular}




\begin{tabular}{|c|c|c|c|}
\hline draw near & $5.2 .10-1 ; 5.4 .6-1$. & earthworm & $9.5-4$. \\
\hline draw out (water) & 7.5.3.1-13. & earwax & $1.3 .6-3$ \\
\hline dread & 6.3.1.3-3. & east & 8.1.6.2-3. \\
\hline dream & $2.3 .2-5$ & east-south-east & 8.1.6.2-4. \\
\hline dregs & 7.7.4.6-9. & Easter & 6.6.4.5-1. \\
\hline dress & $4.1-8$. & eat & $2.1 .2-1 ; 2.1 .2-4$. \\
\hline dribble & $1.3 .7-6$. & eat into & $3.2 .3-5$ \\
\hline dried bananas & 7.7.4.5-2. & eating place & $7.1 .6-5$ \\
\hline drift (with a current) & 7.5.4.1-14. & Echeneis naucrates & $9.9 .23-1$. \\
\hline drift (with the tide) & 7.5.4.1-14. & Echidna nebulosa & $9.9 .6-2$ \\
\hline drift (with the wind) & 7.5.4.1-13. & Echinus sp & $9.12-3 ; 9.12-4$ \\
\hline \multirow[t]{2}{*}{ drill } & \multirow{2}{*}{$\begin{array}{l}\text { 5.4.27-3; 7.11.1-3; } \\
\quad \text { 7.11.1-4. }\end{array}$} & & $9.12-5$. \\
\hline & & echo & $8.2 .2-8$ \\
\hline drink & $2.1 .2-11 ; 7.7 .4 .6-1$. & eczema & $3.2 .3-1$ \\
\hline drink (make s.o. ) & $6.8 \cdot 2.12-2$ & effeminate & 2.4.2.4-9. \\
\hline drink (strong ) & 7.7.4.6-5. & egg (of louse) & $9.2-15$. \\
\hline drinkable & 7.7.4.6-1. & egg (turtle) & $9.10-9$. \\
\hline drinking water & 7.7.4.6-2. & Egghead Parrotfish & 9.9.34-9. \\
\hline drip & $8.1 .5-6$. & eggs (yellow $\sim$, turtle) & $9.10-10$ \\
\hline drizzle & $8.1 .5-5$ & Egretta sacra & $9.1 .3-5$. \\
\hline drool & $1.3 .7-6$ & eight & 7.13.1-9. \\
\hline droop & $5.4 .11-6$. & elbow & $1.5 .1-4$ \\
\hline drop & 7.7.4.4-6. & elderly person & $6.1 .6-13$. \\
\hline drop anchor & 7.5.4.3-4. & elect & $6.5 .2-4$ \\
\hline drought & $8.3 .2-3$ & elephantiasis & $3.4-4$ \\
\hline drought season & $8.1 .3-16$ & eleven & $7.13 .1-12$ \\
\hline drown & $7.5 .5-7$ & eloquence & 6.8.2.1-4. \\
\hline \multirow[t]{2}{*}{ drum } & 6.7.3.1-2; & emasculate & $9.3-17$. \\
\hline & 6.7.3.1-3. & Ember Parrotfish & $9.9 .34-2$. \\
\hline drunk & $2.1 .3-6$ & embers & $7.6 .1-5$ \\
\hline drunkard & $2.1 .3-7$ & embrace & 5.4.2.3-3; \\
\hline dry & $7.8-9 ; 7.12 .5-7$. & & 6.8.3.1-4. \\
\hline dry banana leaf & 10.1.1-11. & emerge & $7.5 .5-6$. \\
\hline dry up & 8.3.2-1. & empty & $7.12 .5-14$. \\
\hline \multirow{2}{*}{$\begin{array}{l}\text { drying structure (copra, } \\
\text { coffee) }\end{array}$} & \multirow[t]{2}{*}{ 7.1.3-3. } & Enchynassa canina & $9.9 .6-1$ \\
\hline & & encircle (fish) & 7.4.1-1. \\
\hline dull & $1.1 .1-2$ & encircled & $5.2 .9-2$ \\
\hline dumb & $3.5-4$ & enclose & 7.1.2-1. \\
\hline Dusky Wrasse & $9.9 .33-14$. & enclosed & $5.2 .9-2$ \\
\hline dust (raise on) (hen) & $9.1 .1-13$ & enclosure & 7.1.2-1. \\
\hline dust (to ) & $7.1 .8-4$ & end & $5.6 .3-1$ \\
\hline dwarf & $3.5-8$ & energy & 6.3.1.3-1. \\
\hline ear & $1.3 .6-1$ & engaged (be $\sim$ ) & $6.1 .2-6$. \\
\hline earrings & $4.3-4$. & enjoy & 2.4.2.4-3. \\
\hline ears (big ) & $1.3 .6-2$ & enlarge & 5.4.18.2-3. \\
\hline earth & $8.2 .1-2$ & enlighten & $6.2 .6-8$ \\
\hline earthquake & $8.2 .1-4$ & enslave & 6.8.4.8-3. \\
\hline
\end{tabular}




enter
enter (a house stealthily)
entrance
envelop
envious
Epibulus insidiator
epilepsy
Epinephelus fasciatus
Epinephelus
$\quad$ hexagonatus
Epinephelus lanceolatus
Epinephelus merra
Epinephelus tauvina
erase
erect
erection (have an $\sim$ )
Erianthus floridulus
eructation
escape (steam, oven
smoke)

Eugena jambos

Euthynnus affinis

even out

evening

evil

eviscerate

ewe

exact

examine

exchange

excrement

expel (when giving birth)

explain

explode into pieces (oven stones)

extend (hand)

eye

eyebrow

eyelash

eyelid

eyetooth

fable

face

facetious

faeces
5.2.6-1.

5.2.6-2.

7.1.5.1-2;

7.1.5.1-5.

$$
\text { 5.4.23-4. }
$$

6.3.2.2-2.

9.9.33-7.

$3.4-2$.

9.9.17-5.

9.9.17-8.

9.9.17-4.

9.9.17-7.

9.9.17-6.

5.4.17-4.

5.1.1-1; 7.1.3-1.

2.4.2.4-5.

10.4.8-11.

2.1.3-2.

7.7.3.1-6.

10.3.5-6.

9.9.46-6.

$7.2 .2-5$.

8.1.3-1.

6.8.1-19.

7.7.2.3-1.

$9.3-21$.

6.2.4-3.

2.4.2.1-4.

6.5.4-5.

2.1.4-6.

6.1.4-5.

6.2.5-6; 6.8.2.4-2; 6.8.2.4-3.

$7.6 .2-7$.

5.4.1-8.

1.3.4-1.

1.3.4-6.

1.3.4-7.

1.3.4-3.

1.3.8-5.

6.7.4-2.

1.3.3-2.

6.8.1-7.

2.1.4-6.
Fagraea berteriana

faint

faint (feel )

fall

fall (backwards)

fall (cause to )

fall (fruit)

fall (upright)

fall down

falling tide

family (extended )

family name

fan

fan (to )

fart

fast (to )

fasten

fat

father

father-in-law

fear

fearful

feasible

feast

feather

fed up

feel

feelings (seat of $\sim$ )

fellow

female

female (plants, insects, crustaceans)

female (which gave birth)

fence

fence off

ferment (to )

fermented (well $\sim$ )

fern

fern (fragrant )

fern (tree )

festival
10.4.3-9; 10.4.4-1;

10.4.4-2.

2.4.1.2-5.

2.4.1.2-6.

5.2.12-2; 5.2.12-6;

5.2.12-7;

5.2.12-9.

5.1.5-5; 5.2.12-5.

5.4.11-4.

$7.2 .6-8$.

5.2.12-8.

5.2.12-3; 5.2.12-4;

$$
\text { 5.2.14-1. }
$$

8.3.4-8.

6.4.1-4.

6.1.1-4.

5.4.24-5.

7.6.1-9.

2.1.4-11.

7.7.4.1-2.

5.4.13-1.

1.1.3-1; 1.1.3-2;

7.7.4.1-3.

$6.4 .3-2$.

6.4.4-2.

6.3.1.3-2;

6.3.1.3-3.

6.3.1.3-4.

5.6.4-6.

6.7.1-2.

9.1.1-2.

6.3.1.4-3.

5.4.1-3.

6.2.2-1.

1.1-1.

9.10-12.

10.4.1-2.

$9.3-29$.

7.1.2-1.

7.1.2-1.

7.7.2.1-5.

7.7.2.1-6.

10.4.11-3.

10.4.11-2.

10.4.11-1.

6.7.5-4. 


\begin{tabular}{|c|c|c|c|}
\hline fetid & 7.7.4.7-6. & fish $($ to $\sim)$ & $7.4-3 ; 7.4-4$ \\
\hline fever & 2.4.1.2-9. & & $7.4-5$. \\
\hline \multirow{2}{*}{$\begin{array}{l}\text { fibre (coconut used as } \\
\text { filter) }\end{array}$} & \multirow[t]{2}{*}{$7.7 .1-17}$. & fish (to $\sim$ ) with a dipnet & $7.4 .6-3$ \\
\hline & & fish (to $\sim$ ) with a line & 7.4.3.1-3. \\
\hline $\begin{array}{l}\text { fibres on shelled coconut } \\
\text { (scrape } \sim \text { ) }\end{array}$ & 7.7.2.2-8. & $\begin{array}{l}\text { fish at night with the } \\
\text { help of a torch }\end{array}$ & $7.4 .6-2$ \\
\hline fibrous lower inner part & $10.4 .2-2$ & fish with several hooks & 7.4.6-4. \\
\hline Fiddler Crab & $9.8-5$. & fisherman & $7.4-3$ \\
\hline \multirow[t]{2}{*}{ fight } & \multirow{2}{*}{$\begin{array}{l}6.8 .4 .4-5 \\
\quad 6.8 .4 .9-1 \\
6.8 .4 .9-2\end{array}$} & $\begin{array}{l}\text { fishing (clouding the } \\
\text { water) }\end{array}$ & $7.4 .4-1$ \\
\hline & & fishing (in the open seas) & $7.4 .6-5$ \\
\hline fight & 7.3.1-1. & fishing (with a net) & 7.4.1-3. \\
\hline fight over & 6.8.4.4-1. & fishing hook & 7.4.3.1-4. \\
\hline \multirow{2}{*}{$\begin{array}{l}\text { Filament-finned } \\
\text { Parrotfish }\end{array}$} & \multirow[t]{2}{*}{$9.9 .34-7$} & fishing line & 7.4.3.1-2. \\
\hline & & fishing net & 7.4.1-5; 7.4.2-1. \\
\hline filariasis & $3.4-3$. & fishing rod & 7.4.3.1-1. \\
\hline file & 7.11.1-1. & Fistularia comersonii & $9.9 .15-1$. \\
\hline file (blade) & 5.4.24-6. & fit & $3.4-2$ \\
\hline file of fishes & $7.4-6$ & five & 7.13.1-6. \\
\hline fill & 7.7.2.1-2. & fix (bait to the hook) & 7.4.3.2-3. \\
\hline fill in & 5.4.21.4-6. & fix a hook & 7.4.3.1-8. \\
\hline fill up & 5.4.21.4-5. & flabby & $1.1 .3-1$ \\
\hline filled by the wind (sail) & 7.5.4.1-12. & Flagtail Grouper & $9.9 .17-3$ \\
\hline fillet (a fish) & 7.7.2.3-2. & flame & 7.6.1-3. \\
\hline filter & 7.7.1-17. & flank & 1.4.1.1-7. \\
\hline filthy & $4.4-9$ & flash of lightning & $8.1 .4-6$ \\
\hline fin & $9.9 .1-10$. & flat & $7.12 .2-10$ \\
\hline \multirow{2}{*}{ fine (s.o.) } & \multirow{2}{*}{$\begin{array}{l}6.8 .4 .8-5 \\
\quad 6.8 .4 .8-8\end{array}$} & flat part of the reef & $9.13 .2-4$ \\
\hline & & flat-nosed & $1.3 .5-2$ \\
\hline \multirow{2}{*}{$\begin{array}{l}\text { fine weather after rain } \\
\text { finger }\end{array}$} & \multirow{2}{*}{$8.1 .5-7}$. & flatten & 5.4.18.2-1; 7.2.2-5. \\
\hline & & flatter & $6.8 .2 .6-3$ \\
\hline finish & $5.2 .15-3 ; 5.6 .3-1$ & flavour & 2.4.2.2-1. \\
\hline fire & $7.6 .1-2 ; 7.6 .2-12$ & flea & $9.2-17$ \\
\hline fire (light a ) & $7.6 .1-4 ; 7.6 .1-8$ & flee & $5.2 .11-1$ \\
\hline fire (put out $\sim$ ) & $7.6 .2-14$ & flesh & $1.2-2 ; 7.7 .2 .1-7$. \\
\hline fire $(s e t \sim t o)$ & $7.6 .2-10$ & flick & $5.4 .16-3$ \\
\hline firebrand & $7.6 .1-6$. & flipper (of shark) & $9.9 .1-12$. \\
\hline firestick & $\begin{array}{l}7.6 .1-6 . \\
7.61-11 \cdot 761-12\end{array}$ & flipper (turtle) & $9.10-5 ; 9.10-6$. \\
\hline firewood & 1.6.1-11; 7.6.1-12. & flock & $9.3-31$. \\
\hline firewood (bundle of $\sim$ ) & $7.6 .2-11$ & flog & $5.4 .28-3$ \\
\hline $\begin{array}{l}\text { firewood (bundle of } \sim) \\
\text { first (the } \sim \text { ) }\end{array}$ & 7.11.3-2. & flood (biblical ) & 6.6.4.5-4. \\
\hline $\begin{array}{l}\text { first (the ) } \\
\text { first born (child) }\end{array}$ & 7.13.2-1. & floor & 7.1.3-7. \\
\hline $\begin{array}{l}\text { first born (child) } \\
\text { fish }\end{array}$ & $6.4 .1-7$. & floorcloth & $7.1 .8-3$ \\
\hline $\begin{array}{l}\text { fish } \\
\text { fish (raw } \sim)\end{array}$ & $9.9 .1-1$ & flow & $8.3 .1-6$. \\
\hline & 7.7.4.4-2. & flower & $10.1 .2-5$ \\
\hline & 9.9.1-7. & Flowerly Flounder & $9.9 .41-1$. \\
\hline fish (threaded ) & $7.4-6$. & flowers (bunch of $\sim$ ) & $10.1 .2-4$ \\
\hline
\end{tabular}




\begin{tabular}{|c|c|c|c|}
\hline flowing tide & 8.3.4-7. & Fringelip Mullet & $9.9 .36-1$. \\
\hline flute (nose $\sim$ ) & 6.7.3.1-4. & frizzy & $1.3 .2-2$ \\
\hline fly & $9.1 .1-7$ & frond (large lower part & 10.4.2-3. \\
\hline fly & $9.2-14$ & of the ) & \\
\hline fly away & $9.1 .1-7$ & frond of coconut tree & $10.4 .2-1$ \\
\hline Flyingfish & $9.9 .11-2$. & front (of the house) & 7.1.6-8. \\
\hline foam & $8.3 .3-4$ & froth (of court-bouillon) & 7.7.3.3-3. \\
\hline fold & $5.4 .23-3 ; 5.4 .23-5$. & fruit (bear ) & $10.1 .3-2$ \\
\hline follow & $5.2 .4-3$ & fruit (cluster of $\sim$ ) & $10.1 .3-3$ \\
\hline fontanel & $1.3 .1-4$ & fruit (net for $\sim$ ) & $7.2 .5-12$ \\
\hline food & 7.7.4.1-1. & fruit season & 7.2.6-1. \\
\hline food (over-cooked $\sim$ ) & 7.7.3.2-1. & fruit stone & $7.2 .3-6$ \\
\hline food parcel (in oven) & 7.7.4.2-1. & fuddled & $2.1 .3-8$ \\
\hline fool (s.o.) & 6.8.2.5-1. & full up & $2.1 .2-8$ \\
\hline fooled $(b e \sim)$ & 6.8.2.5-3. & fun (make $\sim$ of $)$ & 6.8.4.2-3. \\
\hline foot & $1.5 .2-1$ & funnel & 7.7.1-18. \\
\hline \multirow{2}{*}{$\begin{array}{l}\text { foot-stalk and central } \\
\text { vein of coconut palm }\end{array}$} & \multirow[t]{2}{*}{$10.4 .2-4}$. & furious & $6.3 .2 .3-4$ \\
\hline & & furuncle & $3.2 .2-3$ \\
\hline forbid & $6.6 .3-2$ & Galeocerdo Cuvier & $9.9 .2-6$ \\
\hline forbidden & $6.6 .3-3$ & gall & $1.4 .2-6$ \\
\hline force (to do) & 6.8.4.8-1. & gallant & $6.8 .1-6$ \\
\hline fore flipper (turtle) & $9.10-5$. & gallery & 7.1.4-5. \\
\hline forefinger & $1.5 .1-9$ & game & 6.7.1-1; 6.7.1-3; \\
\hline forehead & $1.3 .3-1$ & & 6.7.1-9. \\
\hline foreland & $8.2 .2-6$ & ganglion & $3.2 .2-2$ \\
\hline foreman & $6.5 .2-8 ; 6.8 .5 .5-3$. & garden & $7.2 .3-1$ \\
\hline forewoman & 6.8.5.5-3. & gargle & $3.6-3$. \\
\hline fork & 7.7.1-9. & gather & 5.4.21.2-1; 7.2.5-5. \\
\hline forked & $7.12 .2-4$ & gather (firewood) & $5.4 .21 .4-2$ \\
\hline form (good physical $\sim$ ) & $3.1-2$ & gathering & 6.8.5.1-2; \\
\hline formerly & $8.1 .3-12$ & & 6.8.5.4-2. \\
\hline Forsten's Parrotfish & $9.9 .34-10$ & gecko & $9.4-3$ \\
\hline forty six & 7.13.1-17. & genealogy & $6.4 .1-1 ; 6.4 .1-2$ \\
\hline foster parent & $6.4 .1-10 ; 6.4 .1-11$. & generation & $6.1 .1-11$. \\
\hline founder & 7.5.4.2-5. & generous & 6.5.3.2-1. \\
\hline four & $7.13 .1-5$. & gentle & $6.8 .1-12$ \\
\hline four $(b y \sim)$ & 7.13.3-3. & ghost & $6.6 .2-1 ; 6.6 .2-2$. \\
\hline fracture & $3.1-14$. & giant clam & $9.6 .2-14$ \\
\hline fragrance & $2.4 .2 .2-3$ & Giant Grouper & 9.9.17-4. \\
\hline fragrant fern & $10.4 .11-2$ & Giant Jack & $9.9 .24-6$ \\
\hline Fregata ariel & $9.1 .2-11$. & Giant Moray & $9.9 .6-5$ \\
\hline Fregata minor & $9.1 .2-10$ & giddy & $2.4 .1 .2-6$ \\
\hline fresh & 2.4.1.1-5. & gift (make a $\sim$ ) & $5.4 .4-2$ \\
\hline fresh water & 7.7.4.6-3. & gills & $9.9 .1-8$ \\
\hline friend & 6.3.2.4-2. & ginger & 7.7.2.4-4; 10.2.1-5. \\
\hline frighten & 6.3.1.3-2. & girdle & $4.1-11$ \\
\hline frightful & 6.3.1.3-4. & girl & $6.1 .6-4 ; 6.1 .6-5$. \\
\hline
\end{tabular}




\begin{tabular}{|c|c|c|c|}
\hline give & $\begin{array}{l}\text { 5.4.4-1; 6.5.3.1-1; } \\
\quad 6.5 .4-6\end{array}$ & $\begin{array}{l}\text { gouge } \\
\text { governor }\end{array}$ & $\begin{array}{l}7.11 .1-4 . \\
6.5 .2-6 .\end{array}$ \\
\hline give birth & $6.1 .4-2$ & grab & $6.5 .5-2$ \\
\hline give cover to & 7.1.1-4. & Grammistes sexlineatus & $9.9 .18-1$. \\
\hline glad & 2.4.1.3-4; & granddaughter & $6.4 .3-7$ \\
\hline & 6.3.1.1-2. & grandfather & $6.4 .3-6$ \\
\hline glance & 2.4.2.1-5; 5.5.2.-1. & grandmother & $6.4 .3-5$ \\
\hline glans & 1.4.1.3-2. & grandnephew & $6.4 .3-7$ \\
\hline glass & 7.7.1-22. & grandniece & $6.4 .3-7$ \\
\hline Glasseye & $9.9 .22-1$. & grandparents & $6.4 .3-4$ \\
\hline gleams of sun & 8.1.1-6. & grandson & $6.4 .3-7$ \\
\hline glimpse & 5.5.2.-1. & granulous & $7.12 .5-10$. \\
\hline Globehead Parrotfish & $9.9 .34-5$. & grasp & 5.4.2.5-5; 6.2.5-4. \\
\hline glory & 6.3.1.3-5. & grasshopper & $9.2-21$ \\
\hline gluttonous & $2.1 .2-3$ & grater (coconut $\sim$ ) & 7.7.1-2. \\
\hline gnarled & 7.11.4-6. & grater (tuber $\sim$ ) & 7.7.1-3. \\
\hline gnash teeth & $2.3 .1-6$ & grave & $6.1 .8-6$ \\
\hline Gnathanodon speciosus & $9.9 .24-4$ & gravel & $8.2 .1-5$ \\
\hline $\begin{array}{l}\text { Gnathodentex } \\
\text { aureolineatus }\end{array}$ & $9.9 .27-2$. & Gray Duck & $9.1 .3-7$. \\
\hline go & $5.2 .1-1 ; 5.2 .1-5$. & graze & $5.4 .16-5$ \\
\hline go astray & $5.1 .7-3$. & grease & 7.7.2.1-4. \\
\hline go down & $5.2 .2-2$ & grease (animal $\sim$ ) & 7.7.4.1-3. \\
\hline go fetch & $5.2 .1-2$ & Greasy Grouper & $9.9 .17-6$. \\
\hline go out & $5.2 .6-3$ & Great Barracuda & $9.9 .35-1$. \\
\hline go outside & $5.2 .6-3$ & Great Frigatebird & $9.1 .2-10$. \\
\hline go quickly by & $5.1 .7-4$ & great-grandchildren & $6.4 .3-8$ \\
\hline go round & $5.2 .9-3$ & $\begin{array}{l}\text { great-great- } \\
\text { grandchildren }\end{array}$ & $6.4 .3-9$ \\
\hline go slowly & $5.2 .1-4$ & greedy & $2.1 .2-3 ; 6.3 .2 .2-4$. \\
\hline go to sea & 7.5.4.1-2. & green & $7.12 .4-3$. \\
\hline goat & $9.3-22$ & greet, greeting & 6.8.2.2-1; \\
\hline goatee beard & $9.3-24$. & & 6.8.2.2-6. \\
\hline Gobiidae & $9.9 .38-1$. & grey & $7.12 .4-8$. \\
\hline goby & $9.9 .38-1$. & grey hair & $1.3 .2-5$ \\
\hline goitre & $3.4-7$ & Grey Jobfish & $9.9 .25-2$ \\
\hline Golden Jack & $9.9 .24-4$. & Grey Reef Shark & $9.9 .2-3$ \\
\hline Goldlined Emperor & $9.9 .27-2$ & grill & 7.7.3.2-7. \\
\hline Gomphosus varius & $9.9 .33-18$ & grimy & $4.4-9$. \\
\hline gone off (raw fish, fruit) & 7.7.4.7-3. & grind & 5.4.18.1-4; \\
\hline gonorrhoea & $3.4-14$. & & $5.4 .21 .3-2$ \\
\hline good for nothing & $6.8 .1-3$ & grind teeth & $2.3 .1-6$ \\
\hline good temperature & 2.4.1.1-5. & grip & $5.4 .2 .5-5$ \\
\hline good-looking (man) & $1.1 .2-2$ & groin & 1.4.1.2-7. \\
\hline goose pimples & 2.4.1.1-4. & groove and tongue & 7.11.4-7. \\
\hline gorge & $2.1 .2-4 ; 2.1 .2-7$. & ground & $8.2 .1-2$ \\
\hline gormandize & $2.1 .2-4$ & grounds (grated & 7.7.2.4-11. \\
\hline gospel & 6.6.4.5-7. & coconut $\sim$ ) & \\
\hline
\end{tabular}




\begin{tabular}{|c|c|c|c|}
\hline group & $9.3-31$. & handwash & $7.8-1$ \\
\hline grove (coconut $\sim$ ) & 7.2.3-11. & hang & $5.2 .9-4 ; 5.4 .15-2$. \\
\hline grow & $7.2 .3-8$. & hang (o.'s head) & $5.5 .1-3$ \\
\hline growl & $9.3-3$. & hang lower & $5.5 .1-3$ \\
\hline $\operatorname{grub}($ to $\sim)$ & $7.2 .2-3$. & hanging & $5.4 .15-1$ \\
\hline grudge & 6.8.4.4-7. & happiness & 2.4.1.3-4. \\
\hline grudge (have a $\sim$ ) & 6.3.2.3-3. & harelip & $3.5-7$ \\
\hline grumble & 6.8.4.6-3. & harpoon & 7.4.2-3. \\
\hline grunt & $9.3-3$. & hasten & $5.2 .1-3 ; 5.6 .2-6$. \\
\hline guest & 6.8.3.5-1. & hat & $4.2-1$. \\
\hline Guettarda speciosa & 10.4.3-6; 10.4.3-7. & hatch & $9.1 .1-11$. \\
\hline gulf & $8.2 .2-5$ & have sex & 2.4.2.4-1. \\
\hline gum & 1.3.4-5; 1.3.8-8. & head & $1.3 .1-2$ \\
\hline \multirow[t]{2}{*}{ gummy } & 7.12.5-12; & head (of fish) & $9.9 .1-5$. \\
\hline & 7.12.5-15. & head (turtle) & $9.10-7$ \\
\hline gush out & $8.3 .1-3$. & head for & 7.5.4.1-3. \\
\hline gust (of wind) & 8.1.6.1-3. & headache & $2.4 .1 .2-8$ \\
\hline gut & $1.4 .2-4$. & head-rest & 7.1.7-10. \\
\hline gut (animal, fish) & 7.7.2.3-1. & health & $3.1-1$ \\
\hline guts & 1.4.1.2-6. & healthy & $3.1-2$ \\
\hline guzzle & $2.1 .2-4$ & hear & 2.4.2.3-1. \\
\hline Gygis alba & $9.1 .2-16$ & heart & $1.4 .2-1$ \\
\hline Gymnomuraena zebra & $9.9 .6-3$. & heart (coconut palm) & 10.4.2-9. \\
\hline Gymnosarda unicolor & $9.9 .46-5$. & heartwood & $7.11 .2-3$. \\
\hline \multirow{2}{*}{$\begin{array}{l}\text { Gymnothorax } \\
\text { flavimarginatus }\end{array}$} & \multirow[t]{2}{*}{$9.9 .6-7$} & heat & $7.6 .2-1 ; 7.6 .2-13$. \\
\hline & & heathen & 6.6.4.1-1. \\
\hline Gymnothorax javanicus & $9.9 .6-5$. & heavy & $7.12 .5-9$. \\
\hline Gymnothorax meleagris & $9.9 .6-6$. & heel & $1.5 .2-7$ \\
\hline Gymnothorax undulatus & $9.9 .6-8$. & height & $1.1 .1-3$ \\
\hline haemorrhoids & $3.4-8$. & hello & $6.8 .2 .2-6$. \\
\hline hair & $1.3 .2-1$ & help & $6.8 .3 .2-3$. \\
\hline Halfbeak & $9.9 .11-1$. & Hemigymnus fasciatus & $9.9 .33-16$. \\
\hline half-flower & $10.4 .6-2$ & Heniochus monoceros & $9.9 .31-1$. \\
\hline Halichoeres hortulanus & $9.9 .33-12$. & herd & $9.3-31$ \\
\hline Halichoeres marginatus & $9.9 .33-14$ & Hermit Crab & $9.8-12$ \\
\hline \multirow{2}{*}{$\begin{array}{l}\text { Halichoeres } \\
\text { ornatissimus }\end{array}$} & \multirow[t]{2}{*}{$9.9 .33-15}$. & hernia & $3.1-12$ \\
\hline & & Heterocentratus & $9.12-2$ \\
\hline $\begin{array}{l}\text { Halichoeres } \\
\text { trimaculatus }\end{array}$ & 9.9.33-13. & Heteropriacanthus & $9.9 .22-1$ \\
\hline halo (sun, moon) & 8.1.1-14. & cruentatus & \\
\hline hammer & $7.11 .1-5$. & Heteroscelus incanus & $9.1 .3-3$ \\
\hline hand & $1.5 .1-1$. & hibiscus & $10.4 .6-1$ \\
\hline hand joint & $1.5 .1-3$ & Hibiscus rosa sinensis & $10.4 .6-1$ \\
\hline handle & $7.2 .1-1$ & hiccough & $2.2-9$ \\
\hline handle (basket ) & 7.9.3-2. & hidden & $5.4 .7-3 ; 6.6 .3-1$ \\
\hline handle (knife ) & 7.7.1-15. & hide & $5.4 .7-3 ; 5.4 .7-4$ \\
\hline handle (of paddle) & 7.5.3.1-11. & & $6.6 .3-1$. \\
\hline
\end{tabular}




\begin{tabular}{|c|c|c|c|}
\hline high & $7.12 .3-4$. & house (for bathing) & 7.1.6-4. \\
\hline Highfin Chub & $9.9 .28-10$. & house (front of $\sim$ ) & 7.1.6-8. \\
\hline highly-spiced & 7.7.4.7-9. & house for cooking & 7.1.6-7. \\
\hline hill & 8.2.2-9. & howl & $9.3-5$ \\
\hline Himantura fai & $9.9 .3-1$. & huddle & $5.2 .10-2$ \\
\hline hind flipper (turtle) & $9.10-6$. & huddle up & $5.1 .3-4$. \\
\hline hip & 1.4.1.2-1. & human being & $1.1-1$ \\
\hline Hipposcarus longiceps & $9.9 .34-6$. & humble & $6.3 .2 .1-4 ; 6.8 .1-15$. \\
\hline hit & $5.2 .13-1 ; 5.4 .28-6$. & Humphead Wrasse & $9.9 .33-2$. \\
\hline hit obstacle (ship) & 7.5.4.2-2. & hundred & 7.13.1-18. \\
\hline hither & $5.2 .7-1$ & hunger, hungry & 2.4.1.2-1. \\
\hline hobble & $5.2 .3-6$ & hunting (wild) pigs & 7.3.2-1. \\
\hline hoe & $7.2 .1-7$. & hurricane & 8.1.6.1-6. \\
\hline hold & $\begin{array}{l}\text { 5.4.2.3-1; } \\
\text { 5.4.2.3-9. }\end{array}$ & hurry & $\begin{array}{r}5.2 .1-3 ; 5.2 .11-6 \\
5.6 .2-6 ; 5.6 .2-7\end{array}$ \\
\hline hold out & $5.4 .1-8$. & hurry (be or do in $a \sim$ ) & $5.2 .11-6$ \\
\hline hold s.o. up & 5.4.2.1-7. & husband & $6.1 .1-9$. \\
\hline hold tight & 5.4.2.3-2. & husk (of coconut) & 10.3.2--6. \\
\hline hole & 7.2.2-2. & Hyporhamphus affinis & $9.9 .11-1$. \\
\hline hole (coconut) & 10.3.2-3. & idea & $6.2 .2-2$ \\
\hline hole (dig a $\sim)$ & $7.2 .2-1$ & idle & $6.8 .1-5$ \\
\hline hole (in plaited bamboo) & 7.1.5.2-4. & ignorant & $6.2 .1-3$ \\
\hline hollow & $7.12 .5-2$ & ill & $3.1-3$ \\
\hline hollow out (a canoe) & $7.5 .2-5$ & illuminate & 8.1.1-7. \\
\hline hollow out (a coconut) & $5.4 .26-4$. & immerse & 5.4.18.3-2. \\
\hline hollow trunk & $10.1 .1-15$. & impatience & $6.8 .1-17$ \\
\hline holy & 6.6.4.1-7. & in-laws & $6.4 .4-1$ \\
\hline Homorocoryphus & $9.2-21$. & Indian Mulberry & $10.3 .5-4$ \\
\hline honey & 7.7.4.3-6. & indolent & $6.8 .1-5$ \\
\hline Honeycomb Grouper & $9.9 .17-7$. & infant & $6.1 .6-2$ \\
\hline honour & $6.5 .1-2$ & infection (foot) & $3.2 .2-7$ \\
\hline hook (fishing ) & 7.4.3.1-4. & inform & $6.2 .6-8$ \\
\hline hook (to fish) & 7.4.3.1-5. & Inocarpus fagifer & 7.7.4.3-4. \\
\hline hop & $5.2 .11-5$ & Inocarpus fagiferus & $10.3 .5-3$ \\
\hline hop along & $5.2 .11-5$ & insect & $9.2-1$ \\
\hline hope & 6.3.2.1-2. & insert (hand) & $5.4 .1-6$. \\
\hline horn & $9.3-23$. & insist & 6.8.2.6-1. \\
\hline horse & $9.3-19$. & insult & 6.8.4.7-1. \\
\hline \multirow[t]{3}{*}{ hot } & 2.1.1-3; 2.4.1.1-1; & intelligence & $6.2 .5-1$ \\
\hline & 7.6.2-12; & interpose & $5.4 .12-2$ \\
\hline & 7.7.4.7-9. & interstice (gap in plaited & 7.1.5.2-4. \\
\hline hot (weather) & 8.1.4-10. & bamboo) & \\
\hline house & 7.1.1-1. & intestine & $1.4 .2-4$ \\
\hline house (back of $\sim$ ) & 7.1.6-9. & intoxicated & $2.1 .3-6$ \\
\hline house (cement $\sim$ ) & $7.1 .4-4$ & intrepid & $6.8 .1-4$ \\
\hline \multirow{2}{*}{$\begin{array}{l}\text { house (corrugated } \\
\text { iron } \sim \text { ) }\end{array}$} & $7.1 .4-3$. & invite & 6.8.3.5-2. \\
\hline & & Ipomoea pes-caprae & $10.4 .7-1$ \\
\hline
\end{tabular}




\begin{tabular}{|c|c|c|c|}
\hline iron (clothing) & $7.10-2$ & knead (the po'e) & 7.7.2.1-8. \\
\hline iron (corrugated $\sim$ ) & 7.1.4-3. & knead (with the hand) & 7.7.2.1-9. \\
\hline ironwood tree & $10.4 .4-6$ & knee & $1.5 .2-3$ \\
\hline irritable & $6.8 .1-10$ & kneel & $5.1 .2-2$ \\
\hline irritated & $6.3 \cdot 2 \cdot 3-3$ & knife & 7.7.1-14. \\
\hline irritating & 2.4.1.2-7. & knife handle & 7.7.1-15. \\
\hline island & 8.2.1-1. & knock (on door) & $5.4 .16-4 ; 5.4 .28-7$. \\
\hline island (low coral ) & $8.2 .2-4$ & knock against & $5.2 .13-1$ \\
\hline Island Jack & $9.9 .24-3$. & knock against s.th. (ship) & 7.5.4.2-2. \\
\hline islet & $8.2 .2-4$ & knock down & 5.4.11-4; \\
\hline isolated (be $\sim$ ) & 6.8.5.1-1. & & 5.4.29.5-4. \\
\hline Istiophorus platypterus & $9.9 .46-2$ & knock fruit down with a & $7.2 .5-10$ \\
\hline itching & $3.2 .1-5$ & pole & \\
\hline jaundice & $3.4-13$ & knot & $5.4 .13-2$ \\
\hline javelin & 7.3.1-5. & knotty & $7.11 .4-6$. \\
\hline jaw & $1.3 .8-1$. & know-how & $5.6 .4-2$ \\
\hline jaw articulation & $1.3 .8-9$. & knowledge & 5.6.4-3; 6.2.1-1; \\
\hline jealous & $\begin{array}{l}6.3 \cdot 2 \cdot 2-2 ; \\
6.3 \cdot 2 \cdot 2-3 .\end{array}$ & Kuhlia mugil & $\begin{array}{c}\text { 6.2.1-2. } \\
9.9 .21-1\end{array}$ \\
\hline jellyfish & $9.13 .1-1 ; 9.13 .1-2$. & Kyphosus cinerascens & $9.9 .28-10$ \\
\hline join & $5.4 .13-7$ & Kyphosus vaigiensis & $9.9 .28-9$. \\
\hline & 5.4.21.2-2; & Labroides bicolor & $9.9 .33-22$. \\
\hline & $5.4 .21 .2-5$ & Lactoria cornuta & $9.9 .44-4$. \\
\hline join a group & 6.8.5.1-4. & ladder & $7.1 .2-3$ \\
\hline joint & $1.2-4$ & lament for & 2.4.1.3-9. \\
\hline joke & 2.4.1.3-1; & lamp & 7.1.7-3; 7.1.7-4. \\
\hline & 6.8.2.1-6. & land & $8.2 .1-2$ \\
\hline jostle & $5.2 .11-3$ & landmark & 7.1.1-2. \\
\hline joy & 2.4.1.3-4; & landslide & 8.2.1-10. \\
\hline & 6.3.1.1-2. & landslip & $8.2 .1-10$ \\
\hline jump & $5.2 .11-4 ; 6.3 .1 .2-3$ & language & 2.4.2.3-2. \\
\hline junction & $8.2 .2-2$ & larder & 7.7.4.5-6. \\
\hline Keeled Needlefish & $9.9 .10-1$. & large-leaved plant & $10.2 .1-4$ \\
\hline keep & 5.4.2.3-9. & ressembling taro & \\
\hline keep repeating & $6.8 .2 .11-2$ & lash & $1.3 .4-7 ; 5.4 .28-3$ \\
\hline kick & $5.3-1 ; 5.3-2$ & lash out & $5.3-2$ \\
\hline & 5.4.11-3. & last & $5.1 .6-3$ \\
\hline kidney & $1.4 .2-8$ & last night & $8.1 .3-8$ \\
\hline kill & $5.4 .28-4$ & late (be $\sim)$ & 6.8.5.3-3. \\
\hline kill an animal & 7.3.2-5. & lather & $7.8-3$ \\
\hline king & $6.5 .2-2$ & laugh & 2.4.1.3-1; \\
\hline kinship & $6.4 .1-3$ & & $2.4 .1 .3-2$ \\
\hline kiss & 6.8.3.1-4. & law & $6.5 .1-3$ \\
\hline kitchen & 7.1.6-7. & lay & $5.4 .5-1 ; 5.4 .5-2 ;$ \\
\hline kite & $6.7 .1-5$ & & $9.1 .1-10$ \\
\hline knead & $5.4 .21 .3-3$. & lay (a net) & $7.4 .1-2$ \\
\hline knead (dough) & 7.7.2.1-9. & layer & $7.2 .4-2$ \\
\hline
\end{tabular}




\begin{tabular}{|c|c|c|c|}
\hline lazy & $6.8 .1-3$ & lift & $5.4 .8-1$. \\
\hline lead & 5.2.4-1; 7.3.1-7. & lift s.th. to put it aside & 5.4.2.3-4. \\
\hline leader & $6.5 .2-5$ & lift up & $5.4 .8-2$ \\
\hline leaf & 10.1.1-10. & light & $7.12 .5-8 ; 8.1 .1-6$. \\
\hline leaf (coconut $\sim$ ) & 7.9.2-2. & light (a fire) & 7.6.1-4; 7.6.1-7; \\
\hline leaf (dry banana ) & 10.1.1-11. & & 7.6.1-8. \\
\hline leaf stalk & $10.1 .3-1$ & light (an oil lamp) & 7.6.1-10. \\
\hline leafflower & $10.4 .8-2$ & light (table ) & 7.1.7-4. \\
\hline leak & 7.7.3.1-6. & lightning & $8.1 .4-5$ \\
\hline leak (canoe) & 7.5.4.2-4. & lightning (flash of $\sim$ ) & $8.1 .4-6$. \\
\hline \multirow[t]{2}{*}{ lean } & 1.1.4-1; 5.1.5-1; & like & 6.3.2.4-1. \\
\hline & 5.4.18.4-3. & liking (take $a \sim$ to) & 6.3.2.1-3. \\
\hline lean o.'s back & $5.1 .4-3$ & $\operatorname{limp}$ & $5.2 .3-6$ \\
\hline leaning to one side & $7.12 .2-3$. & Linchia pacifica Gray & $9.12-1$ \\
\hline lean-to roof & 7.1.4-6. & line (fishing ) & 7.4.3.1-2. \\
\hline leap & $5.2 .11-4$ & line up & $5.4 .5-4$ \\
\hline learn & $6.2 .6-1 ; 6.2 .6-7$. & lineage (filiation) & $6.4 .1-6$ \\
\hline Leatherback & 9.9.24-9. & lip & 1.3.7-2; 1.3.7-3. \\
\hline leave & $5.2 .8-3 ; 5.4 .3-3$. & liquid (thick ) & 7.7.4.6-6. \\
\hline leave (take ) & 6.8.2.2-5. & list (to have $\sim$ ) (ship) & 7.5.4.1-10. \\
\hline leave out & 5.4.22.1-3. & listen & $2.4 .2 .3-1$ \\
\hline leaven & 7.7.2.1-5. & little & $1.1 .1-4$ \\
\hline leaves (pandanus $\sim$ ) & 7.9.2-4; 7.9.2-6. & little finger & $1.5 .1-8$ \\
\hline left (to the $\sim$ ) & 7.5.4.1-4. & Little Shearwater & $9.1 .2-3$ \\
\hline leftover food & 7.7.2.4-9. & live & $5.1 .6-3 ; 7.1 .1-3$. \\
\hline $\operatorname{leg}$ & $1.5 .2-1$ & liver & $1.4 .2-5$ \\
\hline legacy & 6.5.3.1-2. & Liza vaigiensis & $9.9 .36-2$ \\
\hline legend & $6.7 .4-2$ & lizard & $9.4-2$ \\
\hline lemon & $10.3 .4-2$ & lobster & $9.8-10$ \\
\hline length (of house) & 7.1.6-13. & local basil & 10.3.1-3. \\
\hline Lepidium bidentatum & $10.4 .8-3$ & lock & $5.4 .30-4$ \\
\hline leprosy & $3.2 .3-4$ & loincloth & $4.1-3$. \\
\hline Lesser Frigatebird & $9.1 .2-11$. & loins & 1.4.1.2-3. \\
\hline Lesser Golden Plover & $9.1 .3-1$ & long (time) & $6.8 .5 .3-2 ; 8.1 .3-10$. \\
\hline letgo & $5.4 .3-2$ & longevity & $6.1 .1-8$ \\
\hline leucorrhoea & $3.2 .3-7$ & Longhorn Cowfish & $9.9 .44-4$. \\
\hline level & 5.4.18.2-2. & Longnose Parrotfish & $9.9 .34-6$. \\
\hline lever & $7.2 .2-4$ & Longspine Cardinalfish & $9.9 .20-1$ \\
\hline lichen (coconut palm ) & $10.4 .9-2$ & look after & $4.1-1$ \\
\hline \multirow{2}{*}{$\begin{array}{l}\text { lichen with long whitish } \\
\text { filaments }\end{array}$} & \multirow[t]{2}{*}{$10.4 .9-1$} & look for & $5.4 .9-1 ; 5.4 .9-2$. \\
\hline & & look & 2.4.2.1-2; 5.5.2-6. \\
\hline lick & $9.3-10$ & loose woman & $6.5 .6-2$ \\
\hline lid & 7.7.1-10. & loosen & $5.4 .3-1$ \\
\hline \multirow[t]{2}{*}{ lie (position) } & \multirow{2}{*}{$\begin{array}{l}\text { 5.1.3-1;5.1.3-2; } \\
\text { 5.1.3-3. }\end{array}$} & lop off & $5.4 .29 .2-5$. \\
\hline & & lose o.'s way & $5.1 .7-3$ \\
\hline lie, deceive & $6.2 .4-5 ; 6.8 .2 .5-2$. & lose shape & $4.1-7$. \\
\hline life & $3.1-1 ; 6.1 .1-7$. & loud & 6.8.2.10-3. \\
\hline
\end{tabular}




\begin{tabular}{|c|c|c|c|}
\hline lounge & $5.1 .4-5$ & many & $7.13 .3-1$. \\
\hline louse & $9.2-18$. & Manybar Goatfish & $9.9 .28-5$ \\
\hline love & 6.3.2.4-1. & Maori Wrasse & $9.9 .33-4$. \\
\hline low tide & $8.3 .4-8$. & marine shell & $9.6 .2-8$ \\
\hline lower back & 1.4.1.2-3. & mark (boundary ) & 7.1.1-2. \\
\hline Lowfin Chub & $9.9 .28-9$. & marriage & $6.1 .2-2$ \\
\hline luck & $7.4-1$. & marrow & $1.2-5$. \\
\hline lucky & $7.4-1$. & marry & $6.1 .2-1$ \\
\hline lumbago & $3.5-2$. & mash & 5.4.18.1-3. \\
\hline lumbar & 1.4.1.2-3. & Masked Bannerfish & $9.9 .31-1$. \\
\hline lump & $3.2 .2-1$ & Masked Booby & $9.1 .2-7$ \\
\hline lumpy & $7.12 .5-5$. & massage & $3.6-2$. \\
\hline lung & $1.4 .2-3$ & master & $6.2 .5-3$ \\
\hline lure & 7.4.3.2-4. & masticate & $2.1 .2-5$ \\
\hline lure (before fishing) & 7.4.3.2-5. & masturbate & 6.8.4.7-4; \\
\hline lure (for dragline & $7.4 .5-1$ & & 2.4.2.4-7. \\
\hline fishing) & & mat & 7.1.7-7. \\
\hline Lutjanus bohar & $9.9 .25-7$. & mat (pandanus $\sim$ ) & 7.9.1-2. \\
\hline Lutjanus fulvus & $9.9 .25-6$. & mat (plaited $\sim$ ) & 7.9.1-1. \\
\hline Lutjanus gibbus & $9.9 .25-5$. & mat (plaited coconut & 7.1.7-8. \\
\hline Lutjanus kasmira & $9.9 .25-3$. & palm) & \\
\hline Lutjanus monostigma & $9.9 .25-4$. & matter & $3.2 .2-5$ \\
\hline luxuriant & 7.2.3-9. & maul & 5.4.29.5-2. \\
\hline Lyretail Grouper & 9.9.17-10. & meal & $6.7 .5-3$ \\
\hline Mackerel Tuna & $9.9 .46-6$. & measure & $7.10-3$. \\
\hline mad & $3.7-2$ & meat (fish, pork, chicken) & 7.7.2.1-7. \\
\hline maggot & $9.5-1 ; 9.5-2$ & meat (tinned $\sim$ ) & 7.7.4.5-3. \\
\hline magician & $6.6 .1-5$ & meet & $5.2 .5-1 ; 5.2 .5-2$ \\
\hline make & 5.6.1-1. & & 6.8.5.1-3. \\
\hline make clear & 6.8.2.4-2. & meeting & 6.8.5.1-2; \\
\hline make fun of & 6.8.4.6-1. & & $6.8 .5 .4-2$ \\
\hline make peace & 6.8.4.9-3. & meeting place & 6.8.5.4-1. \\
\hline make up & 6.8.3.4-5. & Melichthys niger & $9.9 .42-1$. \\
\hline make vanish & 5.4.29.5-3. & Melichthys vidua & $9.9 .42-2$ \\
\hline make water & $2.1 .4-5$ & melon & 7.7.4.3-3; 10.3.6-3. \\
\hline Malay Apple & $10.3 .5-5$ & mend & $7.10-7$. \\
\hline male & $9.10-12$. & mend (nets) & 7.9.5-1. \\
\hline male (plants, insects, & $10.4 .1-1$ & menorrhagia & $1.2-10$ \\
\hline crustaceans) & & menses & $1.2-9$ \\
\hline malicious & $6.8 .1-19$. & meowing & $9.3-9$. \\
\hline $\operatorname{man}$ & $1.1-1 ; 6.1 .1-9$. & merry & $2.1 .3-8$ \\
\hline Mangifera indica L. & $10.3 .6-6$ & midge & $9.2-9$ \\
\hline mango & $10.3 .6-6$. & midwife & $6.1 .4-3$ \\
\hline manner & $6.8 .1-1$ & migraine & $2.4 .1 .2-8$. \\
\hline Manta birostris & $9.9 .5-1$. & milk & 1.4.1.1-5. \\
\hline Manta Ray & $9.9 .5-1$ & milk (add ) & 7.7.2.4-8. \\
\hline Mantis Shrimp & $9.8-13$. & milk (cow, goat) & $9.3-27$. \\
\hline
\end{tabular}




\begin{tabular}{|c|c|c|c|}
\hline milk (pure coconut $\sim$ ) & 7.7.2.4-1. & mourning songs & $6.1 .8-7$. \\
\hline milk tooth & $1.3 .8-3$ & mouse & $9.3-11$ \\
\hline Milkfish & $9.9 .9-1$ & mouth & $1.3 .7-1$ \\
\hline milt & $9.9 .1-3$. & move & $5.1 .6-1 ; 5.4 .5-5$. \\
\hline mime (with hands) & $5.4 .1-1$ & move (the tail) & $9.3-7$. \\
\hline Mimosa pudica & $10.4 .8-8$ & move (when seated) & $5.1 .4-6$ \\
\hline mince & 7.7.2.3-5. & move back & $5.2 .10-3$. \\
\hline mind & $6.2 .2-1$ & move noisily & $5.1 .6-2$ \\
\hline mingle & 5.4.21.3-1. & move over & $5.2 .3-1$ \\
\hline mint & $10.3 .1-5$ & moved & 6.3.1.2-6. \\
\hline miracle (biblical ) & $6.6 .4 .5-5$ & mucosity (babies' $)$ & $1.3 .5-4$ \\
\hline miscarriage & $6.1 .3-4$ & mucus & $2.1 .4-4$ \\
\hline miser, miserly & 6.5.3.2-2. & mud & $8.2 .1-9$ \\
\hline misfortune & 6.3.1.1-5. & muddy & $8.3 .1-5$ \\
\hline miss & $5.2 .9-7$ & muffled voice & $2.4 .2 .3-4$ \\
\hline mist & $8.1 .4-8$ & Mulberry (Indian ) & $10.3 .5-4$ \\
\hline mistake & $6.8 .1-18$ & mulberry (paper ) & $10.4 .3-2$ \\
\hline mistreat & 5.4.29.5-2. & Mulloidichthys & $9.9 .28-1$ \\
\hline \multirow[t]{3}{*}{$\operatorname{mix}$} & 5.4.21.3-1; & flavolineatus & \\
\hline & $\begin{array}{l}\text { 5.4.21.3-2; } \\
\text { 5.4.21.3-3; }\end{array}$ & $\begin{array}{c}\text { Mulloidichthys } \\
\text { vanicolensis }\end{array}$ & $9.9 .28-2$. \\
\hline & 7.7.2.1-1. & multicoloured & $7.12 .4-15$ \\
\hline mix (a paste, cement) & 7.7.2.1-10. & mum & $6.4 .2-1$ \\
\hline modest & 6.3.2.1-4; 6.8.1-15. & Muricidae & $9.6 .2-9$. \\
\hline molar & $1.3 .8-6$ & murmur & 2.4.2.3-9. \\
\hline Monotaxis grandoculis & $9.9 .27-1$. & Musa fehi & $10.2 .2-2$ \\
\hline moo & $9.3-26$. & muscle & $1.2-7$ \\
\hline moody & $6.8 .1-8$. & muscular & $1.1 .2-3 ; 1.1 .2-4$ \\
\hline moon & $8.1 .1-5$ & mushroom & $10.4 .10-1$ \\
\hline Moorish Idol & $9.9 .38-1$ & mute & $3.5-4$ \\
\hline mop & $5.4 .17-1$. & mycosis & $3.2 .1-7$ \\
\hline mop (the floor) & $7.1 .8-3$. & nail & $1.5 .1-10$ \\
\hline Morinda citrifolia & $10.3 .5-4$ & nail & $7.11 .1-6$. \\
\hline Morinda forsteri & 10.4.7-2. & nail down & 7.11.4-4. \\
\hline morning & $8.1 .3-14$ & naked & $4.4-10$ \\
\hline morning (all the $\sim$ ) & $8.1 .3-14$ & name & $6.1 .1-1$ \\
\hline Morning-glory (Beach ) & 10.4.7-1. & nape of the neck & $1.3 .9-4$ \\
\hline mosquito & $9.2-8$ & nappy rash & $3.2 .1-1$ \\
\hline moth & $9.2-13$ & narrow & $7.12 .3-1$. \\
\hline mother & $6.4 .2-1 ; 6.4 .3-3$ & nasal mucus & $1.3 .5-3$ \\
\hline mother-in-law & $6.4 .4-3$ & Naso annulatus & $9.9 .39-5$. \\
\hline mouldy & 7.7.4.7-4. & Naso brevirostris & $9.9 .39-4$. \\
\hline moult & $9.8-8$ & Naso hexacanthus & $9.9 .39-6$. \\
\hline mount & $5.2 .2-1 ; 8.2 .2-9$. & Naso lituratus & $9.9 .39-1$. \\
\hline Mountain Banana & $10.2 .2-2$ & Naso unicornis & $9.9 .39-3$. \\
\hline mourning & $6.1 .8-1$ & Naso vlamingii & $9.9 .39-2$. \\
\hline mourning ceremony & $6.1 .8-2$ & nasty smell & $2.4 .2 .2-4$ \\
\hline
\end{tabular}




\begin{tabular}{|c|c|c|c|}
\hline nasty taste & 7.7.4.7-5. & nothing & 7.13.1-1. \\
\hline nasty & $6.8 .1-19$. & nought & 7.13.1-1. \\
\hline naupaka & $10.4 .6-2$ & numb & 2.4.1.1-9. \\
\hline nausea & 2.4.1.2-11. & numb (with cold) & 2.4.1.1-2. \\
\hline navel & 1.4.1.2-5. & Numenius tahitiensis & $9.1 .3-2$ \\
\hline navigate & $5.2 .1-6$ & numerous & $7.13 .3-1$. \\
\hline neck & $1.3 .9-2$ & nun & $6.6 .4 .3-1$. \\
\hline needle & $7.10-4$ & nurse & $6.1 .5-2 ; 6.1 .5-6$. \\
\hline neigh & $9.3-20$ & oar & 7.5.3.1-9. \\
\hline Neoniphon opercularis & $9.9 .13-1$ & obedient & 6.8.4.8-4. \\
\hline Neoniphon sammara & $9.9 .13-2$. & obese & $1.1 .3-2$ \\
\hline nephew & $6.4 .3-10$ & obey & 2.4.2.3-1; \\
\hline nest & $9.1 .1-9$. & & 6.8.2.12-3. \\
\hline net (fishing ) & 7.4.1-5; 7.4.2-1. & object & $7.12 .1-1$ \\
\hline net (make a $\sim)$ & $7.9 .5-2$. & observe & 2.4.2.1-3. \\
\hline net for fruit & $7.2 .5-12$ & obstinate & $6.8 .1-11$ \\
\hline nets (mend ) & 7.9.5-1. & occiput & $1.3 .9-4$ \\
\hline nettle-rash (fish & $3.2 .1-8$ & ocean & $8.3 .3-5$ \\
\hline intoxication) & & Oceanic Whitetip Shark & $9.9 .2-4$ \\
\hline new & $7.12 .5-1$ & Ocimum basilicum & 10.3.1-3. \\
\hline new-born baby & $6.1 .6-1$ & octopus & $9.7-1$ \\
\hline nibble (at bait) & 7.4.3.1-10. & octopus (head of an ) & $9.7-4$ \\
\hline nice (man) & $1.1 .2-2$ & offer & 6.5.3.1-3. \\
\hline nice smell & $2.4 .2 .2-3$. & offshoot & $7.2 .3-7$ \\
\hline nick-nack & 7.12.1-1. & oil & 7.7.2.1-4. \\
\hline nickname & $6.1 .1-2$ & oil (to ) & 7.7.2.4-5. \\
\hline night & $8.1 .3-15$ & oily & 7.7.2.1-4. \\
\hline night (last ) & $8.1 .3-8$ & old & $8.1 .3-17$ \\
\hline night (whole ) & $8.1 .3-5$ & old man & $6.1 .6-11$. \\
\hline nightmare & $2.3 .2-6$ & old woman & $6.1 .6-12$ \\
\hline nine & 7.13.1-10. & on o.'s own & $6.8 .1-14$ \\
\hline nipple & 1.4.1.1-6. & one & $7.13 .1-2$ \\
\hline nit & $9.2-15$ & one after the other & $7.13 .3-2$ \\
\hline no & 6.8.2.8-8; & one by one & $7.13 .3-2$. \\
\hline & $\begin{array}{l}\text { 6.8.2.8-9; } \\
\text { 6.8.2.8-10 }\end{array}$ & $\begin{array}{l}\text { open } \\
\text { open }\end{array}$ & $\begin{array}{l}6.4 .1-8 . \\
5.4 .30-1\end{array}$ \\
\hline nod & $5.5 .1-4$ & open (by breaking) & $7.2 .5-1$ \\
\hline noisy & 6.8.2.10-3. & open (earth oven) & 7.7.3.1-7. \\
\hline nono & $10.3 .5-4$. & open (o.'s eyes) & $2.3 .2-4$ \\
\hline north & 8.1.6.2-1. & open (o.'s legs) & $5.3-3$. \\
\hline north-east & 8.1.6.2-2. & open sea & $8.3 .3-6$. \\
\hline nose & $1.3 .5-1$. & open side of a canoe & 7.5.3.1-8. \\
\hline \multirow[t]{3}{*}{ not (Negation) } & 6.8.2.8-8; & operculum & $9.6 .1-2$. \\
\hline & 6.8.2.8-9; & orange & 7.12.4-6; 10.3.4-1. \\
\hline & $6.8 .2 .8-10$ & Orangespine Unicornfish & $9.9 .39-1$ \\
\hline notch (deeply) & 7.11.4-9. & orator & 6.8.2.1-3. \\
\hline notch (of wood) & 7.11.4-3. & Orbicular Batfish & $9.9 .29-1$ \\
\hline
\end{tabular}




\begin{tabular}{|c|c|}
\hline order s.o. & 6.8.2.12-1. \\
\hline orgasm & 2.4.2.4-4. \\
\hline Ornate Wrasse & $9.9 .33-15$ \\
\hline orphan & 6.4.1-9. \\
\hline Ostracion cubicus & $9.9 .44-2$ \\
\hline Ostracion meleagris & $9.9 .44-1$. \\
\hline Ostracion whitleyi & $9.9 .44-3$. \\
\hline otitis & $3.1-4$ \\
\hline out of breath (run ) & $2.2-7$ \\
\hline outrigger & 7.5.3.1-1. \\
\hline outrigger side & 7.5.3.1-7. \\
\hline outrun & $5.2 .5-3$ \\
\hline outside & $7.1 .1-5$. \\
\hline oven & 7.7.1-20. \\
\hline $\begin{array}{l}\text { oven (cooking over } \\
\text { earth } \sim)\end{array}$ & 7.7.3.1-8. \\
\hline oven (earth ) & 7.7.3.1-1. \\
\hline oven (Polynesian ) & 7.7.3.1-1. \\
\hline $\begin{array}{c}\text { oven (prepare the } \\
\text { earth } \sim)\end{array}$ & 7.7.3.1-2. \\
\hline oven (stone ) & 7.7.3.1-1. \\
\hline over ripe & 7.2.6-9. \\
\hline over-cooked food & 7.7.3.2-1. \\
\hline overprotect & 6.8.3.2-4. \\
\hline overproud & 6.8.4.2-1. \\
\hline overseer & $6.5 .2-8$ \\
\hline overtake & $5.2 .5-3$ \\
\hline overtaken (be ) & $5.2 .5-3$ \\
\hline own (on o.'s ) & $6.8 .1-14$ \\
\hline$o x$ & $9.3-25$. \\
\hline $\begin{array}{l}\text { Oxycheilinus } \\
\text { unifasciatus }\end{array}$ & $9.9 .33-5$. \\
\hline oyster & $9.6 .2-17 ; 9.6 .2-18$. \\
\hline Pacific Reef Heron & $9.1 .3-5$. \\
\hline pacify (a fight) & 6.8.3.4-4. \\
\hline pack & $9.3-31$ \\
\hline pack up & $5.4 .13-3$ \\
\hline paddle & 7.5.3.1-9. \\
\hline Paddletail Snapper & $9.9 .25-5$. \\
\hline pagan & 6.6.4.1-1. \\
\hline pain & $3.1-10$ \\
\hline pained (be $\sim$ ) & $\begin{array}{l}\text { 6.3.1.1-6; } \\
\text { 6.3.2.3-3. }\end{array}$ \\
\hline painful & $3.1-10$ \\
\hline Palenose Parrotfish & $9.9 .34-4$. \\
\hline palm (of the hand) & $1.5 .1-2$ \\
\hline palm (pandanus ) & $10.4 .4-11$ \\
\hline
\end{tabular}

\begin{tabular}{|c|c|}
\hline palpitate & $1.4 .2-2$ \\
\hline pandanus & 10.4.4-13. \\
\hline $\begin{array}{l}\text { pandanus (edible part of } \\
\text { the drupe) }\end{array}$ & $10.4 .4-10$ \\
\hline $\begin{array}{l}\text { pandanus (male } \\
\text { inflorescence) }\end{array}$ & $10.4 .4-8$ \\
\hline pandanus (roots of $\sim$ ) & $10.4 .4-12$ \\
\hline $\begin{array}{l}\text { pandanus (thornless } \sim \text { ) } \\
\quad \text { (plaiting) }\end{array}$ & $7.9 .2-5$ \\
\hline pandanus (tree, fruit) & 10.4.4-7. \\
\hline pandanus fruit & 10.4.4-7; 10.4.4-9. \\
\hline $\begin{array}{c}\text { pandanus leaf } \\
(\text { smooth } \sim)\end{array}$ & $7.9 .3-7$. \\
\hline pandanus leaves & 7.9.2-4; 7.9.2-6. \\
\hline $\begin{array}{l}\text { pandanus leaves (tear } \\
\text { up } \sim)\end{array}$ & $7.9 .3-3$ \\
\hline pandanus frond & $10.4 .4-11$ \\
\hline pander to s.o.'s whims & 6.8.2.8-3. \\
\hline pant & $2.2-6$ \\
\hline Panther Flounder & $9.9 .41-2$ \\
\hline pantry & 7.7.4.5-6. \\
\hline papaya & $10.3 .5-2$ \\
\hline paper mulberry & $10.4 .3-2$ \\
\hline $\begin{array}{l}\text { Paracirrhites } \\
\text { hemistictus }\end{array}$ & $9.9 .19-2$. \\
\hline paradise & 6.6.4.1-6. \\
\hline paralysed & $3.5-1$ \\
\hline parcel (food , in oven) & 7.7.4.2-1. \\
\hline parched & $7.12 .5-7$. \\
\hline $\begin{array}{l}\text { parents (father, mother, } \\
\text { uncle, aunt) }\end{array}$ & $6.4 .3-1$ \\
\hline parents-in-law & $6.4 .4-1$ \\
\hline pareu & $4.1-3$ \\
\hline parsonage & 6.6.4.2-2. \\
\hline particle & $7.2 .3-4$ \\
\hline parting (in the hair) & $1.3 .2-6$ \\
\hline partition & $7.1 .5 .2-3$. \\
\hline partner & $6.1 .1-10 ; 6.1 .1-9$. \\
\hline Parupeneus ciliatus & $9.9 .28-6$. \\
\hline Parupeneus cyclostomus & $9.9 .28-7$. \\
\hline $\begin{array}{l}\text { Parupeneus } \\
\text { multifasciatus }\end{array}$ & $9.9 .28-5$ \\
\hline $\begin{array}{l}\text { Parupeneus } \\
\text { pleurostigma }\end{array}$ & $9.9 .28-4$ \\
\hline Parupenus barberinus & $9.9 .28-3$. \\
\hline pass & $8.3 .3-3$ \\
\hline pass water & $2.1 .4-5$ \\
\hline past (in the ) & $8.1 .3-12$. \\
\hline
\end{tabular}




\begin{tabular}{|c|c|c|c|}
\hline pastor & 6.6.4.3-3. & pick (fruit) & $7.2 .5-3 ; 7.2 .5-6$ \\
\hline pat & $5.4 .16-1 ; 5.4 .16-2$ & pick at o.'s food & $2.1 .2-2$ \\
\hline patch & $7.10-7$. & pick up & $5.3-6 ; 5.4 .21 .1-1 ;$ \\
\hline patch (on shark's back) & $9.9 .1-11$. & & $5.4 .21 .2-1$ \\
\hline patch $($ taro ) & 10.2.1-3. & pickaxe & $7.11 .1-8$. \\
\hline Patellidae & $9.6 .2-1$ & piece & 7.7.4.2-3. \\
\hline path & $7.1 .2-2$. & pierce & $5.4 .27-2 ; 5.4 .27-4$. \\
\hline pavement (of old houses) & $7.1 .3-2$ & pig & $9.3-15$ \\
\hline pawpaw & $10.3 .5-2$ & pig (scald a dead $\sim$ to & 7.7.2.2-2. \\
\hline pay & $6.5 .4-1$ & scrape off the hair) & \\
\hline pay (a call) & 6.8.3.5-3. & pile up & 5.4.21.2-4; \\
\hline pay attention & $6.8 .1-16$ & & 5.4.21.4-1. \\
\hline peace (make ) & 6.8.4.9-3. & piles & $3.4-8$ \\
\hline peaceful & $6.8 .1-12$ & pillar & $7.1 .2-4$. \\
\hline pearl & $9.6 .1-3$. & pillow & 7.1.7-10. \\
\hline pearl oyster & $9.6 .2-17$ & pimple & $3.2 .1-4$ \\
\hline pebble & $8.2 .1-6$. & Pinctada margarita & $9.6 .2-17$. \\
\hline peck at & $2.1 .2-2$ & pink & $7.12 .4-9$. \\
\hline Pectinidae & $9.6 .2-15$. & Pink Whipray & $9.9 .3-1$ \\
\hline pee & $2.1 .4-5$ & Pinktail Triggerfish & $9.9 .42-2$ \\
\hline peel & 3.2.1-3; 5.4.29.2-3; & pip & $7.2 .3-5$ \\
\hline & 7.7.2.2-6. & piquant & 7.7.4.7-9. \\
\hline peer out & $5.5 .1-2$ & Pisonia grandis & $10.4 .8-9$ \\
\hline peg (clothes ) & $7.8-8$ & pitch (at moorings) & 7.5.4.3-2. \\
\hline pelvis & 1.4.1.2-1. & placate & $6.1 .5-3$ \\
\hline Pempheris oualensis & $9.9 .28-8$. & place & $7.1 .1-6$. \\
\hline Pemphis acidula & $10.4 .3-8$. & place (meeting ) & 6.8.5.4-1. \\
\hline penetrate & 5.4.18.3-1. & placenta & $6.1 .4-6$. \\
\hline penis & 1.4.1.3-1. & plain & $8.2 .2-13$ \\
\hline pepper & $10.3 .1-4$. & plait (surface) & $7.9 .1-4$ \\
\hline $\operatorname{perch}($ to $\sim)$ & $9.1 .1-6$. & plait, plaited & $4.2-3 ; 7.9 .1-3$ \\
\hline perfume & 2.4.2.2-3; 4.4-5. & plank & $7.11 .3-1$. \\
\hline periods & $1.2-9$ & plant (climbing ) & $10.4 .7-2$ \\
\hline \multirow{3}{*}{$\begin{array}{l}\text { Periplaneta americana } \\
\text { perplexed }\end{array}$} & $9.2-12$ & plant (large-leaved & $10.2 .1-4$ \\
\hline & 6.3.1.2-2; & ressembling taro) & \\
\hline & 6.3.1.2-5. & plant (s.th.) & $7.2 .4-4$ \\
\hline perseverance & $6.8 .1-11$ & plant (seagrape) & $10.4 .8-10$ \\
\hline person & $1.1-1$ & plantain & 7.7.4.3-1. \\
\hline perverse & $6.8 .1-19$. & plantation (taro ) & $7.2 .3-12$ \\
\hline Phaeton lepturus & $9.1 .2-5$. & plants (root of $\sim$ ) & $10.1 .1-1$ \\
\hline Phaeton rubricauda & $9.1 .2-6$. & Platax orbicularis & $9.9 .29-1$ \\
\hline phantom & $6.6 .2-2$ & plate & 7.7.1-21. \\
\hline Phyllanthus simplex & $10.4 .8-2$ & platform & $7.1 .3-7$. \\
\hline Phymatosorus spp. & $10.4 .11-3$ & platter & 7.7.1-19. \\
\hline pick (by cutting) & $7.2 .5-8$ & platter (wooden ) & 7.7.1-19. \\
\hline $\begin{array}{l}\text { pick (by pinching } \\
\text { between two fingers) }\end{array}$ & $7.2 .5-7$ & $\begin{array}{l}\text { Platybelone argulus } \\
\text { platyura }\end{array}$ & $9.9 .10-1$ \\
\hline
\end{tabular}




\begin{tabular}{|c|c|c|c|}
\hline play & $6.7 .1-1$ & pound & 5.4.18.1-4. \\
\hline play board games & $6.7 .1-6$ & pounder (stone $\sim$ ) & 7.7.1-11. \\
\hline play bowls & $6.7 .1-8$ & pour & 5.4.21.4-7. \\
\hline play hide and seek & $6.7 .1-7$ & power (supernatural ) & $5.6 .4-4$ \\
\hline playground & $6.7 .5-1$ & praise & 2.4.1.3-5; 6.5.1-1; \\
\hline playing area & $6.7 .5-1$ & & 6.6.4.1-13. \\
\hline pleasant & $8.1 .4-4$ & pray & 6.6.4.3-6. \\
\hline \multirow[t]{2}{*}{ pleasure } & 2.4.2.4-3; & preach & 6.6.4.3-4. \\
\hline & 6.3.1.1-3. & pregnant (be $\sim$ ) & $6.1 .3-1 ; 6.1 .3-2$ \\
\hline Plectropomus laevis & $9.9 .17-11$ & premature baby & $6.1 .4-1$ \\
\hline plentiful & 7.13.3-1. & present (make a $\sim$ ) & $5.4 .4-2$ \\
\hline pluck & 5.4.10-2; 7.7.2.2-1. & preserved (in salt) & 7.7.4.5-4. \\
\hline pluck (flowers) & $7.2 .5-3 ; 7.2 .5-6$ & press & 5.4.18.4-1; \\
\hline plunge into water & 7.5.4.2-7. & & 5.4.18.4-2; \\
\hline (outrigger) & & & $5.4 .18 .4-4$. \\
\hline \multirow{3}{*}{$\begin{array}{l}\text { Pluvialis } \\
\text { dominicana-Pluvialis } \\
\text { fulva }\end{array}$} & $9.1 .3-1$ & prestidigitator & $6.6 .1-5$ \\
\hline & & pretend (to be sick) & $6.2 .4-6$. \\
\hline & & pretty & $1.1 .2-1$ \\
\hline po'e (dessert) & 7.7.4.4-4. & prevent & $5.4 .12-2$ \\
\hline point at & $5.4 .7-2$ & prey on & 7.4.3.2-1. \\
\hline point out & $5.4 .7-1$ & prick & 1.4.1.3-1; 5.4.27-1. \\
\hline pointed & $7.12 .2-9$. & priest & 6.6.4.3-2. \\
\hline poison fish & $7.4 .4-2$ & problems & 6.3.1.1-7. \\
\hline poisoning & $3.1-9$. & problems (have $\sim$ ) & $6.3 .1 .1-5$ \\
\hline \multirow{2}{*}{$\begin{array}{l}\text { pole (for carrying } \\
\text { bananas) }\end{array}$} & $7.2 .1-2$ & Procelsterna cerulea & $9.1 .2-17$. \\
\hline & & produce & $5.6 .1-1$ \\
\hline $\begin{array}{l}\text { pole (knock fruit with } \\
\quad a \sim)\end{array}$ & $7.2 .5-10$ & produce & $7.2 .5-4$ \\
\hline pole (long to knock & $7.2 .1-9$. & prohibit & $6.6 .3-2 ; 6.6 .3-3$ \\
\hline down fruit) & & projecting roof & $7.1 .4-5$ \\
\hline pole for digging & $7.2 .1-3$ & prolific (woman) & $6.1 .1-13$. \\
\hline \multirow[t]{3}{*}{ polish } & $5.4 .17-5$ & promise & 6.8.2.7-1. \\
\hline & 5.4.18.1-1; & promontory & $8.2 .2-6$. \\
\hline & $7.5 .2-6$. & prop & 7.1.5.1-3. \\
\hline Polypodium pustulatum & $10.4 .11-2$ & prop up & $5.6 .2-2$ \\
\hline \multirow[t]{2}{*}{ poor } & 6.5.3.3-2; & prophesy & 6.6.4.3-5. \\
\hline & 6.5.3.3-3. & prostatitis & $3.4-6$ \\
\hline pope & 6.6.4.1-11. & prostitute & $6.5 .6-1$ \\
\hline porpoise & $9.11-1$ & protest & 6.8.4.4-6. \\
\hline port (direction) & 7.5.4.1-4. & protestant & 6.6.4.1-10. \\
\hline port-wine mark & $3.1-5$. & protestant minister & 6.6.4.3-3. \\
\hline portion & 7.7.4.2-2. & protruding ears & $1.3 .6-2$ \\
\hline Portulaca lutea & $10.4 .8-1$ & proud & $6.8 .4 .2-4$ \\
\hline Porzana tabuensis & $9.1 .3-6$. & provoke & 6.8.4.3-1; \\
\hline possessed & $3.7-4$ & & 6.8.4.3-2. \\
\hline post & $7.1 .2-4$. & prune & $5.4 .29 .2-5$ \\
\hline pot & $7.7 .1-8$. & Pseudodax moluccanus & $9.9 .33-19$. \\
\hline potbellied & $1.1 .3-2$ & Pseudosquilla ciliata & $9.8-13$ \\
\hline
\end{tabular}




\begin{tabular}{|c|c|c|c|}
\hline Pteriidae & $9.6 .2-14$. & quiet & 2.4.2.3-11; \\
\hline Pteriidae & $9.6 .2-17$ & & 6.8.2.10-1. \\
\hline Pterocaesio tile & $9.9 .26-1$. & quieten & $6.1 .8-9 ; 6.8 .3 .4-3$. \\
\hline puffed & $2.2-6$ & quite (sea) & $8.3 .4-6$. \\
\hline Puffinus assimilis & $9.1 .2-3$. & rabid & $9.3-12$ \\
\hline Puffinus lherminieri & $9.1 .2-4$ & rabies & $9.3-12$ \\
\hline Puffinus nativitatis & $9.1 .2-2$. & rack & 7.1.7-5. \\
\hline Puffinus pacificus & $9.1 .2-1$. & rafter (of a roof) & 7.1.5.2-2. \\
\hline pull & $\begin{array}{l}5.4 .10-3 ; 5.4 .10-4 ; \\
\quad 5.4 .10-5\end{array}$ & $\begin{array}{l}\text { rain } \\
\text { rain (slanting } \sim \text { ) }\end{array}$ & $\begin{array}{l}8.1 .5-1 \\
8.1 .5-4\end{array}$ \\
\hline $\begin{array}{l}\text { pull (a canoe) ashore or } \\
\text { to the sea }\end{array}$ & 7.5.4.3-3. & $\begin{array}{l}\text { rain and sun } \\
\text { rainbow }\end{array}$ & $\begin{array}{l}8.1 .4-9 . \\
8.1 .1-3\end{array}$ \\
\hline pull back & 2.4.2.4-6. & raise & $5.4 .8-1$ \\
\hline pull off (fruit) & $7.2 .5-9$ & rake & 7.2.1-7. \\
\hline pull up & $5.4 .10-1 ; 5.4 .10-2$ & ram (coprah) & 5.4.21.4-3. \\
\hline pumpkin & $10.3 .6-1$ & ramble & $5.2 .1-8$ \\
\hline punch & $\begin{array}{l}\text { 5.4.28-2; } 5.4 .28-6 ; \\
\quad 6.8 .4 .4-4 .\end{array}$ & $\begin{array}{l}\text { rambling } \\
\text { rancour }\end{array}$ & $\begin{array}{l}3.7-3 . \\
6.3 \cdot 2.2-5\end{array}$ \\
\hline punish & $\begin{array}{l}\text { 6.8.4.8-5; } \\
\text { 6.8.4.8-8. }\end{array}$ & $\begin{array}{l}\text { ransack } \\
\text { rape }\end{array}$ & $\begin{array}{l}\text { 5.4.9-3. } \\
6.8 .4 .10-1\end{array}$ \\
\hline pupil & 1.3.4-4; 6.2.6-5. & & 6.8.4.10-2. \\
\hline purge & $3.6-6$. & rash & $3.2 .1-1 ; 3.2 .1-8$ \\
\hline purple & $7.12 .4-13$. & rasp & 7.7.1-3. \\
\hline purslane & $10.4 .8-1$ & rat & $9.3-11$ \\
\hline pursue & $5.2 .4-4$ & raucous voice & 2.4.2.3-4. \\
\hline pus & $3.2 .2-5$ & raving & $3.7-3$ \\
\hline push & $\begin{array}{l}\text { 5.4.10-5; } 5.4 .11-1 ; \\
\quad 5.4 .11-2\end{array}$ & $\begin{array}{l}\text { raw } \\
\text { raw fish }\end{array}$ & $\begin{array}{l}7.7 .3 .3-2 . \\
7.7 .4 .4-2 .\end{array}$ \\
\hline push (forward) & & reach & $5.2 .8-1$ \\
\hline push (the canoe) & 7.5.4.3-5. & reach for & $5.4 .1-6$ \\
\hline push away & $5.4 .3-3$. & reach land & $5.2 .8-2$ \\
\hline put a curse on & $6.6 .1-1$ & ready (be $\sim)$ & $5.6 .2-4$ \\
\hline put aside & $\begin{array}{l}5.4 .22 .1-2 ; \\
5.4 .2 .3-4\end{array}$ & $\begin{array}{l}\text { ready (food) } \\
\text { rear (of the house) }\end{array}$ & $\begin{array}{l}7.7 .3 .3-4 . \\
7.1 .6-9 .\end{array}$ \\
\hline put o.'s arm around & 5.4.1-7; 6.8.3.1-4. & reason & $6.2 .2-3$ \\
\hline put on (clothes) & $4.1-8$ & $\begin{array}{l}\text { reason (bring s.o. back } \\
\text { to } \sim \text { ) }\end{array}$ & 6.8.3.4-3. \\
\hline put out (fire) & $7.6 .2-14$ & rebound (rain) & $8.1 .5-3$. \\
\hline put the head out to look & $5.5 .1-2$ & receive & $6.5 .4-2$ \\
\hline put the washing out & $7.8-7$ & red & $7.12 .4-11$ \\
\hline put together & $5.4 .5-3 ; 5.4 .6-2$. & Redblotch Surgeonfish & $9.9 .38-5$. \\
\hline puzzled (be $\sim$ ) & $6.3 .1 .2-5$. & Red-footed Booby & $9.1 .2-9$ \\
\hline quadruple & 7.13.3-3. & Red-tailed Tropicbird & $9.1 .2-6$. \\
\hline quake & $8.2 .1-4$ & reed & $10.4 .8-11$. \\
\hline quarrel & 6.8.4.4-5. & reef & 8.3.3-2; 9.13.2-2. \\
\hline queen & $6.5 .2-3$ & reef (flat part of the $\sim$ ) & $9.13 .2-4$ \\
\hline quench (thirst) & 2.4.1.2-4. & refuse & $6.8 .2 .8-5$ \\
\hline
\end{tabular}




\begin{tabular}{|c|c|c|c|}
\hline $\begin{array}{l}\text { reheat (food cooked in } \\
\text { the earth oven) }\end{array}$ & 7.7.3.1-9. & $\begin{array}{l}\text { right } \\
\text { right (to the } \sim)\end{array}$ & $\begin{array}{l}6.2 .4-3 \\
7.5 \cdot 4.1-5\end{array}$ \\
\hline reject & $6.8 .2 .8-5$ & ring & $4.3-2$ \\
\hline rejoice & $6.7 .1-2 ; 6.3 .1 .1-4$. & Ringtail Maori Wrasse & $9.9 .33-5$. \\
\hline $\begin{array}{l}\text { relationship (establish } \\
\text { kinship } \sim \text { ) }\end{array}$ & $6.4 .1-2$ & $\begin{array}{l}\text { rinse } \\
\text { ripe spotted banana }\end{array}$ & $\begin{array}{l}7.8-6 . \\
7.2 .6-4\end{array}$ \\
\hline relative & $6.4 .1-4$ & ripen & $7.2 .6-2$ \\
\hline religion & 6.6.4.1-4. & ripen & $7.2 .6-6$. \\
\hline religious ceremony & $6.7 .5-2$ & ripped & 5.4.29.1-2. \\
\hline religious feast & $6.7 .5-2$ & rise (in the water level) & 8.3.2-2. \\
\hline relish (of food) & 7.7.2.4-12. & rise (moon, sun, stars) & 8.1.2-2. \\
\hline remedy & $3.6-1$ & rising tide & 8.3.4-7. \\
\hline remember & $6.2 .2-4$ & river & 8.3.1-1. \\
\hline remnant & $7.10-6$. & road & $7.1 .2-2 ; 7.1 .2-5$. \\
\hline $\begin{array}{l}\text { remnants of food (stuck } \\
\text { to the dish) }\end{array}$ & 7.7.2.4-9. & $\begin{array}{l}\text { roam } \\
\text { roar with laughter }\end{array}$ & $\begin{array}{l}5.2 .1-8 . \\
2.4 .1 .3-2\end{array}$ \\
\hline remove & $5.4 .11-1$ & rob & $6.5 .5-1$ \\
\hline rent & 5.4.29.1-2. & rock & $6.1 .5-2$ \\
\hline repair nets & 7.9.5-1. & rock (steep ) & $8.2 .2-3$ \\
\hline repeat & 6.8.2.11-1. & roe & $9.9 .1-4$ \\
\hline replete & $2.1 .2-8$ & roll (intr.) & $5.2 .14-1 ; 5.2 .14-2$ \\
\hline reply & $\begin{array}{l}6.8 .2 .3-3 \\
\quad 6.8 .2 .3-4\end{array}$ & $\begin{array}{l}\text { roll (tr.) } \\
\text { roll (ship) }\end{array}$ & $\begin{array}{l}\text { 5.4.23-1; } 5.4 .23-2 . \\
\text { 7.5.4.1-9. }\end{array}$ \\
\hline request & 6.8.2.3-1. & roller $($ sea $\sim)$ & $8.3 .4-2$ \\
\hline $\begin{array}{l}\text { resentment } \\
\text { reside }\end{array}$ & $\begin{array}{l}6.8 .4 .4-7 . \\
5.1 .6-3 ; 7.1 .1-3 .\end{array}$ & $\begin{array}{l}\text { rollers (to pull ashore } \\
\text { small boats) }\end{array}$ & $7.5 .2-2$ \\
\hline respect & 6.5.1-2; 6.8.3.1-2. & rolling & 7.5.4.1-9. \\
\hline rest on & 5.4.18.4-3. & rolls of dry pandanus & $7.9 .2-6$. \\
\hline retiring & 6.3.1.4-4. & leaves & \\
\hline retort & 6.8.2.3-4. & roof & 7.1.5.2-1. \\
\hline retract (foreskin) & 2.4.2.4-6. & roof (lean-to $\sim)$ & $7.1 .4-6$. \\
\hline reveal & $6.2 .6-4$ & roof (made of coconut & $7.1 .4-2$. \\
\hline revenge (take $\sim)$ & 6.8.4.4-9. & leaves) & \\
\hline reverse & $5.4 .19-1$ & roof (projecting $\sim$ ) & $7.1 .4-5$. \\
\hline revolver & 7.3.1-6. & room & $7.1 .6-12$. \\
\hline reward & 6.8.4.8-7. & root (plants) & 10.1.1-1. \\
\hline rheum & $1.3 .4-5$ & roots (banyan $\sim$ ) & $10.1 .1-8$ \\
\hline $\begin{array}{l}\text { Rhinecanthus } \\
\text { rectangulus }\end{array}$ & $9.9 .42-7$. & $\begin{array}{l}\text { roots (pandanus } \sim \text { ) } \\
\text { roots of coconut palms }\end{array}$ & $\begin{array}{l}10.4 .4-12 \\
10.4 .2-8\end{array}$ \\
\hline $\begin{array}{l}\text { Rhinomuraena quaesita } \\
\text { rib }\end{array}$ & $\begin{array}{l}9.9 .6-4 . \\
\text { 1.4.1.1-8; }\end{array}$ & rope & $\begin{array}{l}\text { 7.9.4-1; 7.9.4-2; } \\
\text { 7.9.4-3. }\end{array}$ \\
\hline Ribbon Moray & $9.9 .6-4$ & Rorippa sarmentosa & $10.4 .8-5$ \\
\hline ribs (with visible $\sim$ ) & $1.1 .4-2$ & Rose Apple & $10.3 .5-6$. \\
\hline rice & $10.3 .1-6$. & rosewood & 10.4.3-3. \\
\hline riddle & $6.7 .4-4$ & rotten & 7.2.6-9; 7.7.4.7-2. \\
\hline ridicule & 6.8.4.6-2. & rotten (fish, fruit) & 7.7.4.7-3. \\
\hline rifle & $7.3 .1-6$. & rough-cast & 7.1.3-4. \\
\hline
\end{tabular}




\begin{tabular}{|c|c|}
\hline round & $7.12 .2-5$. \\
\hline round (wind ) & 7.9.4-4. \\
\hline rouse to anger & 6.8.4.3-3. \\
\hline row (a canoe) & 7.5.3.1-9. \\
\hline rub & 5.4.17-3. \\
\hline rub o.'s eyes & $3.6-5$. \\
\hline rudder & 7.5.3.1-12. \\
\hline rule & $6.5 .1-3$. \\
\hline rumble & $2.1 .3-3$. \\
\hline rumble (thunder) & 8.1.4-3. \\
\hline ruminate & $2.1 .2-5$ \\
\hline rummage & 5.4.9-3. \\
\hline run & 5.2.11-2. \\
\hline run (liquid) & 8.3.1-6. \\
\hline run against & 6.8.4.3-4. \\
\hline run aground (ship) & 7.5.4.2-1. \\
\hline run away & 5.2.11-1. \\
\hline run into & $5.2 .5-2$ \\
\hline run through & $5.4 .27-5$. \\
\hline rush & $10.4 .8-11$. \\
\hline rush at & 5.4.11-3. \\
\hline sack & 6.8.5.5-4. \\
\hline sacred & 6.6.4.1-7. \\
\hline sad, sadness & 6.3.1.1-8. \\
\hline sail & $5.2 .1-6$ \\
\hline sail (set $\sim)$ & 7.5.4.1-2. \\
\hline sail (to $\sim$ ) in a tailwind & 7.5.4.1-6. \\
\hline sail against the wind & 7.5.4.1-8. \\
\hline sail canoe & 7.5.1-3. \\
\hline sail down the wind & 7.5.4.1-6. \\
\hline sail in a headwind & 7.5.4.1-8. \\
\hline sail with wind and tide & 7.5.4.1-7. \\
\hline Sailfin Surgeonfish & $9.9 .38-2$. \\
\hline Sailfish & $9.9 .46-2$. \\
\hline salad bowl & 7.7.1-4. \\
\hline saliva & $1.3 .7-5$. \\
\hline salt (preserved in $\sim$ ) & 7.7.4.5-4. \\
\hline salt (to $\sim)$ & 7.7.2.4-3. \\
\hline salted & $2.1 .1-4$ \\
\hline salted (preserved in salt) & 7.7.4.5-4. \\
\hline salty & $2.1 .1-4$ \\
\hline sand & 8.2.1-7. \\
\hline sand lobster & $9.8-11$. \\
\hline sand star & $9.7-5$. \\
\hline sandalwood & $10.4 .3-1$. \\
\hline Sanderling & $9.1 .3-4$. \\
\hline sandy & $7.12 .5-1$ \\
\hline
\end{tabular}

Santalum insulare

sap

sap (breadfruit )

sapwood

Sargocentron diadema

Sargocentron

microstoma

Sargocentron tiere

satisfied

saucepan

saw

say goodbye

say s.o. is in their right

Scaevaola sericea

scald (dead pig)

scale (of fish)

scale (to fish)

scallop

Scalloped Hammerhead

scarcity

scare

Scarus altipinnis

Scarus forsteni

Scarus ghobban

Scarus globiceps

Scarus oviceps

Scarus psittacus

Scarus rubroviolaceus

Scarus schlegeli

scatter

scent

Schizostachyum

glaucifolium

Schlegel's Parrotfish

school (of fish)

Scolopendra

Scolopendra

subspinipes

Scomberoides lysan

scorn

scorn (show sign of $\sim$ )

Scorpaenopsis diabolus

Scott's Wrasse

scrape (dead pig)
10.4.3-1.

10.1.1-12.

10.2.3-2.

7.11.2-2.

9.9.13-4.

9.9.13-5.

9.9.13-3.

2.1.2-8.

7.7.1-8.

7.11.1-2.

6.8.2.2-5.

6.8.2.8-3.

10.4.6-2.

7.7.2.2-2.

9.9.1-9.

7.7.2.2-7.

9.6.2-15.

9.9.2-7.

8.1.3-13.

6.3.1.3-2.

9.9.34-7.

9.9.34-10.

9.9.34-3.

9.9.34-5.

9.9.34-9.

9.9.34-4.

9.9.34-2.

9.9.34-8.

5.4.22-1; 5.4.22-2;

5.4.23-7.

2.4.2.2-1;

2.4.2.2-2; 4.4-5.

$10.4 .8-12$.

9.9.34-8.

9.9.1-2.

$9.2-20$.

9.2-19.

9.9.24-9.

6.8.4.2-3.

6.8.4.7-5.

9.9.16-1.

9.9.33-8.

7.7.2.2-2. 


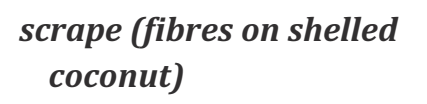

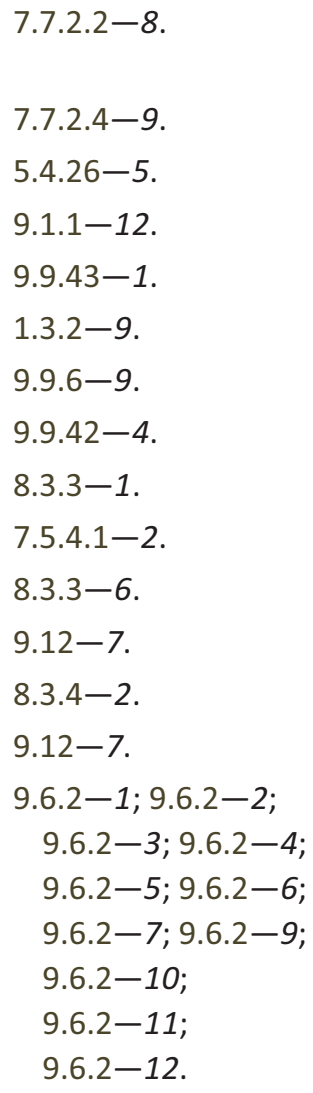

servant, serve

6.5.2-7.

set (in good order)

5.6.2-3.

set (sun, star)

8.1.2-5.

set aside

set fire to

set o.'s teeth on edge

5.4.22.1-1.

7.6.1-8; 7.6.2-10.

set out (hot stones in the oven)

set sail

set square

2.4.1.2-10.

7.7.3.1-4.

7.5.4.1-2.

7.11.1-9.

seven

sever

7.13.1-8.

5.4.29.2-1.

7.10-1.

sew

sex (have )

shadow

shake

shake (cloth)

shake the head

shallow

shallows

shapeless

2.4.2.4-1.

8.1.2-3.

5.4.24-1; 5.4.24-2;

5.4.24-3.

5.4.24-4.

5.5.1-5.

$7.12 .5-16$.

8.2.2-7.

4.1-7.

5.4.22.2-1;

5.4.22.2-4.

share

shark

5.4.22.2-2;

5.4.22.2-5.

9.9.2-1.

sharp

sharpen

shave

shavings (wood )

she, her

sheath of coconut blossoms

sheep

$7.12 .5-4$.

5.4.24-6; 7.11.4-1.

4.4-3.

7.11.4-2.

6.8.6-5; 6.8.6-6;

$$
\text { 6.8.6-7. }
$$

10.4.2-5.

$9.3-21$.

7.1.7-5.

9.6.1-1.

7.1.1-4.

7.1.4-1.

5.4.5-5.

1.5.2-5.

5.4.2.2-3; 7.5.1-1.

5.2.13-2.

4.1-14.

2.1.4-6; 2.1.4-8; 6.8.4.7-4. 


\begin{tabular}{|c|c|}
\hline shoot with a bow & 7.3.1-9. \\
\hline shooting star & $8.1 .1-11$. \\
\hline shore & $8.2 .2-10$ \\
\hline short & 1.1.1-4. \\
\hline Shortnose Unicornfish & $9.9 .39-4$. \\
\hline shotgun & 7.3.1-6. \\
\hline shoulder & 1.4.1.1-2. \\
\hline shout & 6.8.2.2-3. \\
\hline shove away & $5.4 .11-1$ \\
\hline shovel & $7.2 .1-5$ \\
\hline show & $5.4 .7-1 ; 6.2 .6-4$ \\
\hline show off & 6.8.4.3-6. \\
\hline show the way & $5.2 .4-2$ \\
\hline shower & $8.1 .5-2$. \\
\hline shred & 5.4.18.1-4. \\
\hline shrimp (river $\sim$ ) & $9.8-9$ \\
\hline $\begin{array}{l}\text { shrimps (catch river } \\
\quad \text { with net) }\end{array}$ & 7.4.1-4. \\
\hline $\begin{array}{l}\text { shrivel up (during } \\
\text { cooking) }\end{array}$ & 7.7.3.3-5. \\
\hline shroud & $5.4 .7-6$. \\
\hline shy & 6.3.1.4-4. \\
\hline sick & $3.1-3 ; 2.1 .4-1$ \\
\hline side & 1.4.1.1-7. \\
\hline side (of a canoe) & 7.5.3.1-6. \\
\hline side (of a canoe) & 7.5.3.1-7. \\
\hline side (of a canoe) & 7.5.3.1-8. \\
\hline side (of a house) & $7.1 .6-2$. \\
\hline Sidespot Goatfish & $9.9 .28-4$. \\
\hline sideways (walk ) & $5.2 .3-5$ \\
\hline Siganus argenteus & $9.9 .40-1$. \\
\hline Siganus spinus & $9.9 .40-2$. \\
\hline silence & 6.8.2.10-2. \\
\hline silent & $\begin{array}{l}\text { 2.4.2.3-11; } \\
\quad \text { 6.8.2.10-2. }\end{array}$ \\
\hline Silvertip Shark & $9.9 .2-5$ \\
\hline Silvery Rabbitfish & $9.9 .40-1$. \\
\hline $\sin$ & 6.6.4.6-2. \\
\hline sing & 6.7.3.2-1. \\
\hline singe (dead pig) & 7.7.2.2-3. \\
\hline single woman & $6.1 .1-5$ \\
\hline $\sin k$ & 7.5.4.2-5. \\
\hline sink into & 5.4.18.3-1. \\
\hline $\begin{array}{l}\text { sinking (the sun starts } \\
\quad \text { its } \sim \text { ) }\end{array}$ & $8.1 .2-4$ \\
\hline $\operatorname{sip}$ & $2.1 .2-12$. \\
\hline sister (older) & $6.4 .3-14$. \\
\hline
\end{tabular}

\begin{tabular}{|c|c|}
\hline sister (younger) & $6.4 .3-13$. \\
\hline sister-in-law & $6.4 .4-5$ \\
\hline sit & $5.1 .4-1$ \\
\hline sit cross-legged & $5.1 .4-2$ \\
\hline sit on (egg) & $9.1 .1-8$. \\
\hline sit on o.'s heels & $5.1 .4-4$ \\
\hline six & $7.13 .1-7$. \\
\hline Sixbar Wrasse & $9.9 .33-20$ \\
\hline Sixline Soapfish & $9.9 .18-1$. \\
\hline Sixspot Grouper & $9.9 .17-2$. \\
\hline size & $1.1 .1-3$ \\
\hline skeleton & $1.2-3$ \\
\hline skid & $5.2 .12-1$. \\
\hline skilful & $5.6 .4-2$ \\
\hline skilfulness & $6.2 .1-1$ \\
\hline skim & 7.7.1-13. \\
\hline skin & $1.2-1$ \\
\hline skin eruption & $3.2 .1-2$ \\
\hline skip & $6.7 .1-4$ \\
\hline skirt & $4.1-4 ; 4.1-13$. \\
\hline skull & $1.3 .1-1$ \\
\hline sky & $8.1 .1-1$ \\
\hline sky (cloudless $\sim$ ) & $8.1 .1-8$ \\
\hline slander & 5.4.29.5-1. \\
\hline slanting rain & $8.1 .5-4$ \\
\hline slate-pencil urchin & $9.12-2$ \\
\hline slave & 6.8.4.9-7. \\
\hline slaver & $1.3 .7-6$. \\
\hline slay & $5.4 .28-4$ \\
\hline sleep & $2.3 .1-1 ; 2.3 .1-4$ \\
\hline sleeping place & $7.1 .6-6$. \\
\hline sleeplessness & $2.3 .2-3$ \\
\hline sleepy (feel ) & $2.3 .1-2$ \\
\hline Slender Suckerfish & $9.9 .23-1$. \\
\hline slide & $5.2 .12-1$ \\
\hline slide over & $5.2 .3-1$ \\
\hline slim & $1.1 .4-1$ \\
\hline slimy & $7.12 .5-12$. \\
\hline sling & 7.3.1-3. \\
\hline Slingjaw Wrasse & $9.9 .33-7$. \\
\hline slip & $7.2 .4-1$ \\
\hline slippery & $5.2 .12-1$ \\
\hline slobber & $1.3 .7-6$. \\
\hline slope & $8.2 .2-12$. \\
\hline slough & $9.8-8$ \\
\hline smack & $5.4 .28-1$ \\
\hline small & $1.1 .1-4 ; 7.12 .3-3$ \\
\hline
\end{tabular}




small pieces
Smallmouth Squirrelfish
smallpox
Smalltooth jobfish
smart
smear
smell

smell of fish
smelly
smile
smog
smoke
smooth
smooth
smooth (pandanus leaf)
snake
snap
snare
sneak
sneeze
Snooty Wrasse
snore
snot
snout
soak
soak
soap
sob

socket

soft (on the verge of rottenness)

soften

Solanum anthropophagorum

sole (of foot)

solitary

son

son-in-law

song

soothsayer

Sooty Tern

sorcerer

sore throat

sorrow

soul

$$
\begin{aligned}
& \text { 7.7.2.3-3. } \\
& 9.9 .13-5 . \\
& 3.4-12 . \\
& 9.9 .25-1 . \\
& 6.2 .5-1 \\
& 7.1 .3-4 \\
& 2.4 .2 .2-1 \\
& \text { 2.4.2.2-2; } \\
& \text { 2.4.2.2-8. }
\end{aligned}
$$

2.4.2.2-5.

2.1.1-7.

2.4.1.3-3.

8.1.4-8.

7.6.2-3.

7.12.2-8.

5.4.17-5.

7.9.3-7.

9.4-1.

5.4.29.3-1.

7.3.2-3.

6.8.4.5-1.

$2.2-8$.

9.9.33-6.

2.3.1-7.

1.3.5-3.

1.3.5-1.

5.4.18.3-3.

$7.8-4$.

$7.8-2$

2.4.1.3-7.

1.3.4-8.

7.7.4.7-2.

5.4.21.3-4.

10.3.1-4.

1.5.2-8.

6.8.1-14.

6.4.3-10.

6.4.4-6.

6.7.3.2-1.

6.6.1-2.

9.1.2-12.

6.6.1-4.

$3.4-10$.

6.3.1.1-6.

6.6.2-5. sour

2.1.1-5.

south

8.1.6.2-5.

south-south-west

8.1.6.2-7.

sow (seeds)

7.2.3-10.

space (leave a $\sim$ )

5.4.6-2.

spade

7.2.1-5.

Spadefish

9.9.29-1.

spark (to cause )

7.6.2-5.

sparkle

8.1.1-2.

sparks (shower of $\sim$ ) $\quad 7.6 .2-4$.

spasm (have a )

5.1.6-2.

spathe of coconut palm

flowers

spawn

$9.9 .1-4$

speak

2.4.2.3-6.

speak ill of s.o.

6.8.4.6-4.

speak out loud

2.4.2.3-5.

speaker

6.8.2.1-3.

spear

$7.3 .1-5 ; 7.4 .2-3$.

speckled

1.1.2-7.

Spectacled (gray-backed) 9.1.2-13.

Tern

speech

2.4.2.3-6.

speech (make $a \sim$ ) $\quad$ 6.8.2.1-3.

spend (money)

6.5.4-4.

sperm

spherical

2.4.2.4-8.

7.12.2-5.

Sphyraena qenie 9.9.35-2.

Sphyranea barracuda $\quad 9.9 .35-1$.

Sphyrna lewini $\quad 9.9 .2-7$.

spicy

2.1.1-3; 7.7.4.7-9.

spider

spider web

9.2-3.

9.2-4.

7.12.2-1.

spilt

spinal column

$1.2-6$.

spinal cord

$1.2-5$.

spine

$1.2-6$.

spinster

6.1.1-5.

Spiny Rabbitfish

9.9.40-2.

spit

2.1.4-3.

spittle

1.3.7-5.

splice

5.4.13-7.

split

5.4.22.2-3.

split (a fruit)

5.4.29.4-2.

split (wood, fish)

5.4.29.4-1.

spoil

5.4.29.5-1.

Spondias dulcis

10.3.5-5. 


\begin{tabular}{|c|c|c|c|}
\hline Spondylidae & $9.6 .2-18$. & Starry Puffer & $9.9 .45-1$. \\
\hline Spondylus varians & $9.6 .2-18$. & Starspotted Grouper & $9.9 .17-8$. \\
\hline spoon & 7.7.1-12. & start & 6.3.1.2-3. \\
\hline spot & $7.1 .1-6$ & statue & $6.6 .2-4$ \\
\hline Spotfin Squirrelfish & $9.9 .13-2$ & stature & $1.1 .1-3$ \\
\hline Spotless Crake & $9.1 .3-6$. & stay & $5.1 .6-3$. \\
\hline spotted & $1.1 .2-7$ & stay (to maintain & 7.1.5.1-3. \\
\hline Spotted Boxfish & $9.9 .44-1$. & windows open) & \\
\hline Spotted Eagle Ray & $9.9 .4-1$ & stay awake & $2.3 .2-2$ \\
\hline Spotted Hawkfish & $9.9 .19-2$. & steal & $6.5 .5-1$ \\
\hline Spotted Puffer & $9.9 .45-2$ & stealthily (enter a & $5.2 .6-2$ \\
\hline Spotted Surgeonfish & $9.9 .38-8$. & house ) & \\
\hline sprain & $3.1-13$ & steam & 7.6.2-3. \\
\hline spray (water) & 7.2.4-3. & steam (to food) & 7.7.3.2-6. \\
\hline spread & $5.4 .23-6$ & steep rock & $8.2 .2-3$ \\
\hline & 7.7.2.1-12. & Steephead Parrotfish & $9.9 .34-1$. \\
\hline spring & $8.3 .1-4$ & steer (by the stars) & 7.5.4.1-1. \\
\hline spronged spear & $7.4 .2-3$ & stem & $10.1 .1-13$ \\
\hline sprout & $7.2 .3-3 ; 7.2 .3-7$ & stem (ship) & 7.5.3.2-2. \\
\hline sprout (to $\sim)$ & $7.2 .3-2$ & stem (yam, vanilla) & $10.2 .1-1$ \\
\hline spurt & 8.3.1-3. & step back & $5.2 .10-3$ \\
\hline spy upon & $2.4 .2 .1-6$ & step over & $5.3-4$ \\
\hline squall (of wind) & 8.1.6.1-3. & sterile & 6.1.1-12. \\
\hline squama & $3.2 .1-6$ & stern (canoe $\sim$ ) & 7.5.3.1-5. \\
\hline square & $7.11 .1-9$. & Sterna fuscata & $9.1 .2-12$ \\
\hline Squaretail Mullet & $9.9 .36-2$. & Sterna lunata & $9.1 .2-13$. \\
\hline squat & $5.1 .2-1$ & stick & 5.4.21.2-2. \\
\hline squeeze & 5.4.18.4-2. & stick (for lighting fire) & 7.6.1-11. \\
\hline squeeze out & $3.2 .2-6$ & stick (for lighting fire) & $7.6 .1-12$ \\
\hline squill & $9.8-13$. & stick for digging & 7.2.1-3. \\
\hline squint & $3.3-3$. & stick to & $9.6 .1-4$ \\
\hline stack & 5.4.21.4-1. & sticky & $7.12 .5-12$ \\
\hline stagger & $5.2 .1-9 ; 5.2 .3-5$ & still & 2.4.2.3-11. \\
\hline stairs & 7.1.2-3. & still green (fruit) & $7.2 .6-5$ \\
\hline stalk & $10.1 .3-1$ & stilts & 6.7.1-10. \\
\hline stalk & $10.2 .1-1$ & stingray & $9.9 .3-1$. \\
\hline stalk (coconut blossoms) & $10.4 .2-7$ & stink & 2.4.2.2-4; \\
\hline stammer & $2.4 .2 .3-8$ & & 2.4.2.2-6; \\
\hline stamp & $5.2 .1-7$ & & 2.4.2.2-7. \\
\hline stand with hands akimbo & $5.1 .1-3$ & stinking & 7.7.4.7-6. \\
\hline standing & $5.1 .1-1$ & stir & 7.7.2.1-1. \\
\hline star & $8.1 .1-10$ & stir (paste, liquid) & 7.7.2.1-1. \\
\hline star (shooting ) & $8.1 .1-11$. & Stocky Hawkfish & $9.9 .19-1$. \\
\hline starboard & 7.5.4.1-5. & stoky & $1.1 .2-5$ \\
\hline stare at & 2.4.2.1-4. & stone & $8.2 .1-8$ \\
\hline starfish & $9.12-1 ; 9.12-6$. & stone (fruit $\sim$ ) & $7.2 .3-6$. \\
\hline Starry Moray & $9.9 .6-2$ & stone wall & $7.1 .3-5$. \\
\hline
\end{tabular}




\begin{tabular}{|c|c|c|c|}
\hline Stonefish & $9.9 .16-2$. & sugar $($ to $\sim)$ & 7.7.2.4-7. \\
\hline stoop & $5.1 .5-2$ & Sugar Apple & $10.3 .6-4$ \\
\hline \multirow[t]{4}{*}{ stop } & $5.2 .15-1 ; 5.2 .15-2$ & sugarcane & $10.2 .4-1$ \\
\hline & 5.4.12-1; & Sula dactylatra & $9.1 .2-7$ \\
\hline & 5.4.2.3-9; & Sula leucogaster & $9.1 .2-8$. \\
\hline & $6.8 .5 .5-2$ & Sula sula & $9.1 .2-9$. \\
\hline stopper & 7.7.1-7. & sulky & $6.8 .1-8$ \\
\hline story & $6.7 .4-2 ; 6.7 .4-5$ & sultry weather & $7.6 .2-13$. \\
\hline strabismus & $3.3-3$ & sun & $8.1 .1-12$. \\
\hline straight & $7.12 .2-2$. & sun (gleams of $\sim$ ) & $8.1 .1-6$. \\
\hline straight (hair) & $1.3 .2-4$ & sun and rain & $8.1 .4-9$ \\
\hline straighten out & $5.4 .8-3$ & sun-dried (fish, octopus) & 7.7.4.5-5. \\
\hline strangle & $5.4 .18 .4-6$ & sunny & 8.1.1-12. \\
\hline stream & $8.3 .1-1$ & supernatural power & $5.6 .4-4$ \\
\hline strengthen & $5.6 .2-2$ & support s.o. & 5.4.2.1-7. \\
\hline stress (put the $\sim$ on) & 6.8.2.6-1. & surfing & $7.5 .5-4$ \\
\hline stretch (a net) & 7.4.1-2. & surge & $8.3 .4-4 ; 8.3 .4-5$. \\
\hline stretch out & $5.1 .3-1 ; 5.4 .1-8$ & Surge Wrasse & $9.9 .33-21$ \\
\hline stretch out o.'s legs & $5.1 .3-5 ; 5.3-5$ & surname & $6.1 .1-4$ \\
\hline stride over & $5.3-4$ & surprised & 6.3.1.2-3. \\
\hline strike (a bell) & $5.4 .28-5$. & surrounded & $5.2 .9-2$ \\
\hline strike (a fish) & 7.4.3.1-7. & suspended & $5.4 .15-1$ \\
\hline string & $5.4 .14-1$ & swallow & $2.1 .2-7$ \\
\hline string game & $6.7 .1-3$. & swap & $6.5 .4-5$ \\
\hline striped & $7.12 .4-14$. & swear & 6.8.2.7-2. \\
\hline Striped Marlin & $9.9 .46-3$. & swearword & 6.8.4.7-3. \\
\hline Striped Surgeonfish & $9.9 .38-6$. & sweat & $2.1 .4-10$ \\
\hline Striped Triggerfish & $9.9 .42-5$ & sweep & $7.1 .8-2$ \\
\hline stroke & $5.4 .16-2 ; 5.4 .1-4$ & sweet potato & $10.2 .1-7$ \\
\hline Strombidae & $9.6 .2-4$. & sweet potato & 10.2.2-1. \\
\hline strong & 1.1.1-2; 1.1.2-4; & sweeten (with sugar) & 7.7.2.4-7. \\
\hline & $5.6 .4-5$ & swell & $8.3 .4-5$ \\
\hline strong drink & 7.7.4.6-5. & swell (to ) & $7.12 .5-11$. \\
\hline struggle & 6.8.4.9-1. & swelling & $3.2 .3-2 ; 3.2 .3-3$. \\
\hline stubble (burn ) & $7.2 .2-7$ & swim & $7.5 .5-1$ \\
\hline stubborn & $6.8 .1-11$ & swim backstroke & $7.5 .5-2$ \\
\hline stump & 10.1.1-14. & swimming (go ) & $7.5 .5-3$. \\
\hline stupid & $3.7-2$ & swing & $5.2 .9-4$ \\
\hline stutter & $2.4 .2 .3-8$ & swollen & $3.2 .3-2 ; 7.12 .5-11$. \\
\hline submissive & 6.8.4.8-4; 6.8.1-9. & swollen gland & $3.2 .2-2$ \\
\hline successful & $5.6 .3-2$ & Synanceia verrucosa & $9.9 .16-2$. \\
\hline suck & $2.1 .2-9 ; 6.1 .5-1$. & Synodus variegatus & $9.9 .7-1$ \\
\hline sucker & $9.7-3$ & tabernacle & 6.6.4.4-2. \\
\hline Sufflamen bursa & $9.9 .42-4$. & table light & 7.1.7-4. \\
\hline Sufflamen fraenatum & $9.9 .42-3$. & Tacca pinnatifida & $10.4 .8-6$ \\
\hline suffocate & $2.2-5$ & Tacca pinnatifida & $10.4 .8-7$ \\
\hline sugar & 7.7.4.3-7. & tack & 7.5.4.1-11. \\
\hline
\end{tabular}




\begin{tabular}{|c|c|c|c|}
\hline Tahitian Arrow-root & $10.4 .8-6 ; 10.4 .8-7$. & tell (story) & $6.7 .4-3$. \\
\hline Tahitian Squirrelfish & $9.9 .13-3$. & telltale & 6.8.4.5-1. \\
\hline tail & $9.1 .1-3 ; 9.3-6$. & temperature (good $\sim)$ & 2.4.1.1-5. \\
\hline tail (fish) & $9.9 .1-7$. & temple (traditional ) & $6.6 .2-3$ \\
\hline tail (turtle $\sim$ ) & $9.10-3$. & tempt & 6.8.2.6-2. \\
\hline take & $5.4 .2 .3-1$. & ten & 7.13.1-11. \\
\hline take aim & $7.3 .2-2$. & tendon & $1.2-7$. \\
\hline take away & 5.4.2.2-2; 5.4.5-6. & & \\
\hline take by force & $6.5 .5-2 ; 6.8 .4 .10-1$. & tentacle & $9.7-2$. \\
\hline take care of & $\begin{array}{l}4.1-1 ; 6.8 .3 .3-1 \\
6.8 .3 .3-2\end{array}$ & $\begin{array}{l}\text { Ierminalla catappa } \\
\text { testament }\end{array}$ & $\begin{array}{l}10.3 .5-1 . \\
6.6 .4 .5-3 .\end{array}$ \\
\hline take off & $5.4 .5-6$. & testicles & 1.4.1.3-3. \\
\hline take off (clothes) & $4.1-9$. & tether & 5.4.13-9. \\
\hline take out (feathers, hair) & 7.7.2.2-1. & Tetrapturus audax & $9.9 .46-3$. \\
\hline take out (inside pulp of & 7.7.2.2-5. & tetter & $3.2 .3-1$ \\
\hline coconut) & & Thalassoma hardwicke & $9.9 .33-20$. \\
\hline taken in (be $\sim)$ & 6.8.2.5-3. & Thalassoma purpureum & $9.9 .33-21$. \\
\hline talk & $\begin{array}{l}\text { 6.8.2.1-1; } \\
\quad 6.8 \cdot 2.9-1 .\end{array}$ & thank you & 6.3.1.1-1. \\
\hline talk through o.'s nose & 2.4.2.3-7. & Thespesia populnea & $10.4 .3-3$ \\
\hline talkative & $\begin{array}{l}\text { 6.8.2.1-5; } \\
\text { 6.8.2.9-2. }\end{array}$ & $\begin{array}{l}\text { thick } \\
\text { thick (liquid) }\end{array}$ & $\begin{array}{l}7.12 .2-6 . \\
7.7 .4 .6-6 .\end{array}$ \\
\hline tall (man) & $1.1 .1-1$ & thick-lipped & $1.3 .7-3$. \\
\hline talons (bird) & $9.1 .1-4$ & thief & $6.5 .5-1$ \\
\hline tame & 6.8.2.12-4. & thigh & $1.5 .2-2$ \\
\hline tangle up & 7.4.3.1-9. & thin & $7.12 .2-7$ \\
\hline tap & $\begin{array}{l}\text { 5.4.16-1; 5.4.16-4; } \\
\text { 5.4.24-1. }\end{array}$ & $\begin{array}{l}\text { thin } \\
\text { thing }\end{array}$ & $\begin{array}{l}1.1 .4-1 . \\
7.12 .1-1 .\end{array}$ \\
\hline tapa & $7.10-8$. & thing & $7.12 .1-1$ \\
\hline taro & $10.2 .1-2$ & Тाпк & $6.2 .2-3$ \\
\hline taro patch & $10.2 .1-3$ & thirsty $(b e \sim)$ & 2.4.1.2-3. \\
\hline taro plantation & $7.2 .3-12$ & thirty five & 7.13.1-16. \\
\hline tart & $2.1 .1-5$ & thorn & $10.1 .1-5$ \\
\hline $\begin{array}{l}\text { taste (bad ) (bruised } \\
\text { breadfruit) }\end{array}$ & 7.7.4.7-8. & $\begin{array}{l}\text { thorny } \\
\text { Thorny Oyster }\end{array}$ & $\begin{array}{l}7.12 .2-11 . \\
9.6 .2-18 .\end{array}$ \\
\hline taste (having a nasty $\sim$ ) & 7.7.4.7-5. & thought & $6.2 .2-2$ \\
\hline tasteless & 7.7.4.7-7. & thousand & 7.13.1-19. \\
\hline tattoo & $4.3-5$. & thread & 5.4.14-1; 7.4-6; \\
\hline teach & $6.2 .6-1$ & & $7.10-9$. \\
\hline teacher & $6.2 .5-3$ & threaded fish & $7.4-6$. \\
\hline team & $6.8 .5 .1-5$. & threadworm & $9.5-3$. \\
\hline tear (s.th.) & 5.4.29.1-1. & three & 7.13.1-4. \\
\hline $\begin{array}{l}\text { tear up (pandanus } \\
\text { leaves) }\end{array}$ & 7.9.3-3. & three (by ) & $7.13 .3-2$ \\
\hline tears & 2.4.1.3-8. & Threespot Wrasse & $9.9 .33-13$. \\
\hline tease & $\begin{array}{l}\text { 6.3.2.3-2; } \\
\text { 6.8.4.1-1. }\end{array}$ & $\begin{array}{l}\text { throat } \\
\text { throat infection }\end{array}$ & $\begin{array}{l}1.3 .9-2 . \\
3.4-10 .\end{array}$ \\
\hline teeth & $1.3 .8-2$ & throb & $1.4 .2-2$ \\
\hline
\end{tabular}




\begin{tabular}{|c|c|c|c|}
\hline throw & $5.4 .25-1 ; 5.4 .25-2 ;$ & totter & $5.2 .1-9$ \\
\hline & $5.4 .25-3$ & touch & $5.4 .1-3$ \\
\hline & $5.4 .25-5$ & touch (with nose) & 2.4.2.2-2. \\
\hline & $5.4 .25-6$ & touch land & 7.5.4.3-1. \\
\hline throw away & $5.4 .25-4 ; 5.4 .3-3$ & touched & 6.3.1.2-6. \\
\hline throw stones & $7.3 .2-4$. & Tournefortia argentea & $10.4 .4-3$ \\
\hline thumb & $1.5 .1-7$ & tow & $5.4 .10-6$. \\
\hline thunder & $8.1 .4-2 ; 8.1 .4-3$. & toward speaker & $5.2 .7-1$ \\
\hline tibia & $1.5 .2-5$ & trail & $5.4 .10-6$ \\
\hline tide (rising ) & 8.3.4-7. & trample & $5.2 .1-7$ \\
\hline tidy & $5.4 .21 .1-2$ & translator & $6.7 .4-1$ \\
\hline tidy (be ) & $5.6 .2-3$ & transpierce & $5.4 .27-5$ \\
\hline tie & $\begin{array}{l}5.4 .13-2 ; 5.4 .13-3 \\
\quad 5.4 .13-4\end{array}$ & Trapeziidae & $9.6 .2-19$. \\
\hline Tiger Moray & $9.9 .6-9$. & Trapezium oblongum & 9.6.2-19. \\
\hline Tiger Shark & $9.9 .2-6$ & travel & $5.2 .1-6$ \\
\hline tight & $\begin{array}{l}2.1 .3-8 ; 4.1-6 \\
7.12 .3-1\end{array}$ & $\begin{array}{l}\text { tread } \\
\text { tree (ironwood ) }\end{array}$ & $\begin{array}{l}5.2 .1-7 \\
10.4 .4-6\end{array}$ \\
\hline tighten (fishing line) & 7.4.3.1-6. & tree fern & 10.4.11-1. \\
\hline tighten (screw) & 5.4.18.4-5. & trick & $6.8 .2 .5-1$ \\
\hline tiller & 7.5.3.1-12. & Tricoloured Fusilier & $9.9 .26-1$. \\
\hline tilt & $5.4 .11-5 ; 5.4 .20-1$. & Tridacna maxima & $9.6 .2-14$. \\
\hline timber & 10.1.1-9. & trim & $\begin{array}{l}\text { 5.4.18.2-2; } \\
\text { 5.4.29.2-5. }\end{array}$ \\
\hline time & $8.1 .3-4$ & trip s.o. up & $5.3-7$ \\
\hline timid & $6.8 .1-9$ & triple & $7.13 .3-2$ \\
\hline tinned meat & 7.7.4.5-3. & Tripletail Wrasse & $9.9 .33-3$. \\
\hline tiny & $7.12 .3-3$. & triplets & $6.1 .4-8$ \\
\hline tipsy & $2.1 .3-8$. & tropical almond & $10.3 .5-1$ \\
\hline tired, tiredness & 2.4.1.1-8. & trouble (be in $\sim$ ) & 6.3.1.1-5; \\
\hline tiresome & 2.4.1.2-7. & & 6.3.1.1-7. \\
\hline Titan Triggerfish & $9.9 .42-6$. & true & $6.2 .4-1$ \\
\hline toast $($ to $\sim)$ & 7.7.3.2-4. & Trumpetfish & $9.9 .14-1$. \\
\hline tobacco & $10.4 .5-1$ & trunk & 7.1.7-1; 10.1.1-14. \\
\hline toe & $1.5 .1-6$ & trust & 6.3.2.1-1; 6.5.1-2; \\
\hline toilet & 7.1.6-3. & & 6.8.3.1-3. \\
\hline tomb & $6.1 .8-6$. & truth & $6.2 .4-2$ \\
\hline tomorrow & $8.1 .3-2$. & try & $5.6 .4-8$ \\
\hline tomorrow night & $8.1 .3-3$ & tuberculosis & $3.4-11$ \\
\hline tongue & $1.3 .7-4$ & tuft & $1.3 .2-7$ \\
\hline tongue and groove & 7.11.4-7. & tumble down & $5.2 .12-4$ \\
\hline tonsillitis & $3.4-10$ & Turbinidae & $9.6 .2-2$ \\
\hline tool (to extract copra) & 7.2.1-6. & turn & $5.2 .7-2$ \\
\hline tooth & $1.3 .8-2$ & turn & 8.1.6.1-5. \\
\hline top & 5.4.29.2-5. & turn (ship) & 7.5.4.1-11. \\
\hline topple over & $5.2 .14-1$ & turn against & 6.8.4.4-8. \\
\hline topsy-turvy & $5.2 .9-1$ & turn aside & $5.2 .9-7$. \\
\hline torn & 5.4.29.1-2. & turn away & $5.2 .9-6$. \\
\hline
\end{tabular}




\begin{tabular}{|c|c|c|c|}
\hline turn back & $5.2 .7-3$ & unripe (fruit) & $7.2 .6-5$ \\
\hline turn inside out (clothes) & $4.1-12$ & untie & $5.4 .13-11$ \\
\hline turn into & $5.4 .19-4$. & unveil & $5.4 .30-2 ; 6.2 .6-4$ \\
\hline turn o.'s back upon & 5.2.9-1. & uphill slope & $8.2 .2-12$ \\
\hline turn over & 5.4.19-1. & uproot & $5.4 .10-1$ \\
\hline turn over (ship) & 7.5.4.2-6. & upside down & $5.2 .9-1$ \\
\hline turn over in o.'s sleep & $2.3 .1-5$ & urchin (sea $\sim$ ) & $9.12-4 ; 9.12-5$. \\
\hline turn round & $5.1 .1-2 ; 5.2 .9-3$. & urinate & $2.1 .4-5$ \\
\hline turn s.th. onto its side & $5.4 .19-3$. & us & 6.8.6-8; 6.8.6-9; \\
\hline turn the head & $5.5 .1-1$ & & $6.8 .6-12$ \\
\hline turn towards & $5.1 .5-4$ & & $6.8 .6-13$ \\
\hline turn upside down & $5.4 .19-2$ & vagina & 1.4.1.3-6. \\
\hline turtle & $9.10-1 ; 9.10-13$. & vain & 6.8.4.2-2. \\
\hline turtle (flipper of $a \sim$ ) & $9.10-5 ; 9.10-6$. & valley & $8.2 .2-1$ \\
\hline turtle (head of a ) & $9.10-7$ & vanilla stem & $10.2 .1-1$ \\
\hline turtle (young ) & $9.10-11$ & vanquished (be $\sim$ ) & $6.8 .2 .12-4$. \\
\hline turtle beak & $9.10-8$. & Variegated lizardfish & 9.9.7-1. \\
\hline turtle belly & $9.10-4$. & variola & $3.4-12$ \\
\hline turtle egg & $9.10-9$. & Variola louti & $9.9 .17-10$. \\
\hline turtle tail & $9.10-3$. & veer & $5.2 .7-2 ; 5.2 .7-3$ \\
\hline twelve & 7.13.1-13. & vegetable garden & $7.2 .3-1$ \\
\hline twenty & $7.13 .1-14$. & vegetable season & 7.2.6-1. \\
\hline twenty three & 7.13.1-15. & vein & $1.2-7$ \\
\hline twice $($ do $\sim)$ & $7.13 .3-3$. & verandah & 7.1.4-5. \\
\hline twig & $6.2 .5-4 ; 10.1 .1-2$ & vessel & 7.5.1-1. \\
\hline twin & $6.1 .4-7$ & vexation & 6.3.1.1-6. \\
\hline twinkle & $8.1 .1-2$ & vicarage & 6.6.4.2-2. \\
\hline two & $7.13 .1-3$. & violence $($ do $\sim)$ & 5.4.29.5-2. \\
\hline two $(b y \sim)$ & 7.13.3-1. & Viper Moray & $9.9 .6-1$. \\
\hline tyrannize & 6.8.4.8-2. & viscous & $7.12 .5-15$. \\
\hline ugly & $1.1 .2-6$ & visit & $5.2 .5-1$ \\
\hline unbind & $5.4 .13-11$ & visitor & $6.8 .3 .5-1$. \\
\hline uncle & $6.4 .3-2$ & Vlaming's unicornfish & $9.9 .39-2$. \\
\hline unclean & $4.4-8$. & voice & $2.4 .2 .3-2$ \\
\hline uncover & $5.4 .30-2$ & voice (charming ) & $2.4 .2 .3-3$. \\
\hline uncover (earth oven) & 7.7.3.1-7. & voice (high ) & 2.4.2.3-5. \\
\hline Undalated Moray & $9.9 .6-8$. & voluminous & $1.1 .1-2$ \\
\hline undercooked & 7.7.3.3-1. & vomit & $2.1 .4-1$ \\
\hline undercut (fillet of fish) & 7.7.2.3-2. & vulva & 1.4.1.3-4. \\
\hline understand & $6.2 .5-4$ & wafer (communion $\sim$ ) & $6.6 .4 .5-6$. \\
\hline undress & $4.1-9$. & Wahoo & $9.9 .46-4$ \\
\hline unerring & $2.1 .1-5$ & wait & $6.8 .5 .3-4$ \\
\hline unfasten & $5.4 .13-11$ & wake up & 2.3.2-1. \\
\hline unfold & $4.1-10 ; 5.4 .23-6$ & walk & $5.2 .1-5 ; 5.2 .1-8$. \\
\hline unite & 5.4.21.2-3. & walk (cautiously) & $5.2 .3-4$ \\
\hline unlucky & $7.4-2$ & walk (crooked) & $5.2 .3-5$ \\
\hline unrespectful (be ) & 6.3.2.1-5. & walk (in a zigzag) & $5.2 .7-4$ \\
\hline
\end{tabular}




\begin{tabular}{|c|c|c|c|}
\hline walk (knock-kneed) & $5.2 .3-3$ & weather (fine $\sim$ after & $8.1 .5-7$. \\
\hline walk (sideways) & $5.2 .3-5$ & rain) & \\
\hline wall & 7.1.5.2-3. & weave (surface) & 7.9.1-4. \\
\hline wall (stone $\sim$ ) & 7.1.3-5. & web (spider ) & $9.2-4$ \\
\hline wander & $5.2 .1-8$ & wedge & 7.1.3-9. \\
\hline wandering (of the mind) & $3.7-3$. & wedge up & $7.1 .3-9$. \\
\hline Wandering Tattler & $9.1 .3-3$. & $\begin{array}{r}\text { Wedge-tailed } \\
\text { Shearwater }\end{array}$ & $9.1 .2-1$ \\
\hline want & $6.2 .3-1 ; 6.3 .2 .2-1$ & Wedge Triggerfish & $9.9 .42-7$. \\
\hline wanton & $6.5 .6-2$. & weep & 2.4.1.3-6. \\
\hline war & 7.3.1-1. & welcome & 6.8.3.5-2; \\
\hline warmth & 7.6.2-13. & & 6.8.3.5-4. \\
\hline warning & $6.8 .3 .2-1$ & well-built & $1.1 .2-5$ \\
\hline warp (wood) & $7.11 .4-8$. & west & 8.1.6.2-8. \\
\hline wart & $3.2 .3-6$ & west-north-west & 8.1.6.2-9. \\
\hline wash & $4.4-1 ; 5.4 .17-2$. & wet & 7.12.5-17; \\
\hline wash (by hand) & $7.8-1$. & & $7.12 .5-18$ \\
\hline wash o.'s mouth out & $3.6-4$ & wetnurse & $6.1 .5-6$. \\
\hline washing (put the $\sim$ out) & $7.8-7$ & whale & $9.11-2$. \\
\hline wasp & $9.2-6$ & whatsit & 7.12.1-1. \\
\hline waste & $\begin{array}{l}\text { 3.2.3-5; 6.5.4-3; } \\
\quad 6.5 .4-4 .\end{array}$ & $\begin{array}{l}\text { whirlwind } \\
\text { white }\end{array}$ & $\begin{array}{l}8.1 .6 .1-6 . \\
7.12 .4-10 .\end{array}$ \\
\hline \multirow[t]{2}{*}{ watch } & 2.4.2.1-2; & white (skin) & $1.1 .2-7$ \\
\hline & 2.4.2.1-6. & Whitecheek Surgeonfish & $9.9 .38-4$ \\
\hline watch (on the $\sim$ ) & $2.3 .2-2$ & Whitelined Goatfish & $9.9 .28-6$. \\
\hline watch over s.o. & 6.8.3.3-2. & Whitemargin & $9.9 .39-5$. \\
\hline watchful & $2.3 .2-2$ & Unicornfish & \\
\hline water (add ) & 7.7.2.4-6. & Whitemouth Moray & $9.9 .6-6$. \\
\hline water (dip into ) & $7.8-4$ & Whitespotted Puffer & $9.9 .45-3$ \\
\hline water (drinking ) & 7.7.4.6-2. & White-tailed Tropicbird & $9.1 .2-5$ \\
\hline water (fresh ) & 7.7.4.6-3. & Whitleys Boxfish & $9.9 .44-3$. \\
\hline \multirow{2}{*}{$\begin{array}{l}\text { water (in the bottom of } \\
\text { a boat) }\end{array}$} & 7.5.4.2-3. & wide-eyed & $1.3 .4-2$ \\
\hline & & widen & 5.4.18.2-4. \\
\hline water (to ) & $7.2 .4-3$ & widow & $6.1 .8-3$ \\
\hline watermelon & 7.7.4.3-5; 10.3.6-2. & widower & $6.1 .8-4$ \\
\hline wattle (fish ) & $9.9 .1-14$. & width & 7.1.6-1. \\
\hline wave & 8.3.4-1; 8.3.4-4. & wife & $6.1 .1-10$. \\
\hline wave to & $5.4 .12-3$ & wild & $9.3-13$. \\
\hline wavelet & 8.3.4-3. & will & $6.2 .3-1$ \\
\hline wavy & $1.3 .2-3$ & wilt & $10.1 .2-2$ \\
\hline way & $6.8 .1-1 ; 7.1 .2-2$. & win & 6.8.4.9-5. \\
\hline weak & 2.4.1.1-7; 6.8.1-5. & wind & 8.1.6.1-1. \\
\hline \multirow[t]{3}{*}{ we, us } & $6.8 .6-8 ; \quad 6.8 .6-9 ;$ & wind (bring up ) & $2.1 .3-2$ \\
\hline & $6.8 .6-12$ & wind (east ) & 8.1.6.2-3. \\
\hline & $6.8 .6-13$ & wind (east-south-east ) & 8.1.6.2-4. \\
\hline wealthy & 6.5.3.3-1. & wind (north ) & 8.1.6.2-1. \\
\hline weapon & $7.3 .1-4$. & wind (north-east ) & 8.1.6.2-2. \\
\hline
\end{tabular}




\begin{tabular}{|c|c|c|c|}
\hline wind (sail against the $\sim$ ) & 7.5.4.1-8. & wring a piece of wet & $7.8-5$. \\
\hline wind (sail down the $\sim$ ) & 7.5.4.1-6. & clothing & \\
\hline wind (south $\sim)$ & $8.1 .6 .2-5$. & wrinkled & $1.1 .2-6$. \\
\hline wind (south-south- & 8.1.6.2-7. & wrist & $1.5 .1-3$. \\
\hline west ) & & write & $6.2 .6-2$ \\
\hline wind (squall of $\sim$ ) & 8.1.6.1-3. & wrong & $6.2 .4-4 ; 6.2 .6-6$. \\
\hline wind $($ to $\sim)$ round & $7.9 .4-4$ & yam & 10.2.1-6. \\
\hline wind (west ) & 8.1.6.2-8. & yam stem & 10.2.1-1. \\
\hline wind (west-north-west) & 8.1.6.2-9. & yawn & $2.3 .1-3$. \\
\hline window & 7.1.5.1-4. & year & $8.1 .3-11$. \\
\hline windy & 2.4.1.1-6. & yeast & 7.7.2.1-5. \\
\hline wine & 7.7.4.6-8. & yellow & 7.12.4-7. \\
\hline wipe & 5.4.17-1; 5.4.17-2. & yellow (fruit, turn ) & $7.2 .6-6 ; 7.2 .6-7$ \\
\hline wipe o.'s nose & $2.1 .4-2$ & Yellow Boxfish & $9.9 .44-2$ \\
\hline wish & 6.3.2.2-1. & yellow eggs (turtle) & $9.10-10$ \\
\hline wither & $10.1 .2-2$ & Yellow Margin Moray & $9.9 .6-7$. \\
\hline witness & 6.8.2.4-4. & Yellowfin Goatfish & $9.9 .28-2$ \\
\hline wolf down & $2.1 .2-7$ & Yellowfin Surgeonfish & $9.9 .38-7$ \\
\hline woman & $6.1 .1-10$. & Yellow-margined & $9.9 .25-6 ; 9.9 .25-7$. \\
\hline wood & $7.11 .2-1$. & Snapper & \\
\hline wood & 10.1.1-9. & Yellowsaddle Goatfish & $9.9 .28-7$. \\
\hline wood (notch of $\sim)$ & $7.11 .4-3$. & Yellowstripe Goatfish & $9.9 .28-1$ \\
\hline $\operatorname{wood}(\operatorname{rose} \sim)$ & 10.4.3-3. & Yellowtail Coris & $9.9 .33-11$ \\
\hline wood (sandal ) & 10.4.3-1. & yelp & $9.3-5$ \\
\hline wood (soft core) & $7.11 .2-3$. & yes & $6.8 .2 .8-6$ \\
\hline wood shavings & $7.11 .4-2$. & & 6.8.2.8-7. \\
\hline woolly & $1.3 .2-2$ & yesterday & $8.1 .3-7$. \\
\hline word & $\begin{array}{r}2.4 .2 .3-10 \\
2.4 \cdot 2.3-6\end{array}$ & you & $\begin{array}{l}6.8 .6-4 ; \quad 6.8 .6-10 ; \\
\quad 6.8 .6-14 .\end{array}$ \\
\hline work & 5.6.1-2; 6.8.5.5-1. & young & $6.1 .6-9$ \\
\hline worm & $9.5-2$ & young (bird) & $9.1 .1-5$. \\
\hline worm (fruit ) & $9.5-1$ & young (of animal) & $9.3-30$. \\
\hline worried (be $\sim$ ) & 6.8.4.1-3. & young boy & $6.1 .6-6$. \\
\hline worries & 6.3.1.1-7. & young girl & $6.1 .6-4$ \\
\hline wrap & $5.4 .23-4$ & young man & $6.1 .6-7$. \\
\hline wrap up & $5.4 .13-3$. & Zanclus cornatus & $9.9 .38-1$. \\
\hline wreath & $4.3-1$ & Zebra Moray & $9.9 .6-3$. \\
\hline wrench & $3.1-13$ & Zebrasoma veliferum & $9.9 .38-2$. \\
\hline wriggle & $9.9 .1-13$. & zigzag & $5.2 .7-4$. \\
\hline wring (clothes) & $5.4 .20-2$ & & \\
\hline
\end{tabular}

